\title{
Identification and Expression of New Unspecific Peroxygenases - Recent Advances, Challenges and Opportunities
}

\author{
Alina Kinner, Katrin Rosenthal and Stephan Lütz* \\ Chair for Bioprocess Engineering, Department of Biochemical and Chemical Engineering, TU Dortmund University, \\ Dortmund, Germany
}

OPEN ACCESS

Edited by:

Aitao Li,

Hubei University, China

Reviewed by:

Wuyuan Zhang,

Tianjin Institute of Industrial Biotechnology, Chinese Academy

of Sciences, China

Martin Weissenborn,

Leibniz Institute of Plant Biochemistry,

Germany

*Correspondence:

Stephan Lütz

stephan.luetz@tu-dortmund.de

Specialty section:

This article was submitted to

Bioprocess Engineering,

a section of the journal

Frontiers in Bioengineering and

Biotechnology

Received: 05 May 2021

Accepted: 09 June 2021

Published: 07 July 2021

Citation:

Kinner A, Rosenthal K and Lütz S (2021) Identification and Expression of New Unspecific Peroxygenases -

Recent Advances, Challenges and Opportunities.

Front. Bioeng. Biotechnol. 9:705630.

doi: 10.3389/fbioe.2021.705630
In 2004, the fungal heme-thiolate enzyme subfamily of unspecific peroxygenases (UPOs) was first described in the basidiomycete Agrocybe aegerita. As UPOs naturally catalyze a broad range of oxidative transformations by using hydrogen peroxide as electron acceptor and thus possess a great application potential, they have been extensively studied in recent years. However, despite their versatility to catalyze challenging selective oxyfunctionalizations, the availability of UPOs for potential biotechnological applications is restricted. Particularly limiting are the identification of novel natural biocatalysts, their production, and the description of their properties. It is hence of great interest to further characterize the enzyme subfamily as well as to identify promising new candidates. Therefore, this review provides an overview of the state of the art in identification, expression, and screening approaches of fungal UPOs, challenges associated with current protein production and screening strategies, as well as potential solutions and opportunities.

Keywords: unspecific peroxygenase (UPO), oxyfunctionalization, fungal enzyme, heme enzyme, genome mining, heterologous expression, high-throughput screening

\section{INTRODUCTION}

The selective oxyfunctionalization of organic molecules is one of the most challenging tasks in synthetic chemistry. Biotransformations are used in the pharmaceutical industry to replace complex chemical syntheses, e.g., to obtain drug metabolites for pharmacological activity and toxicity studies (Rosenthal and Lütz, 2018). Especially, the large group of heme enzymes harbors versatile biocatalysts, which oxidize non-activated $\mathrm{C}-\mathrm{H}$ bonds regio- and stereospecifically under mild reaction conditions (Schmitz et al., 2019a). In 2004, a new type of heme-thiolate enzyme with mono(per)oxygenase activity was discovered in the basidiomycetous fungus Agrocybe aegerita catalyzing a broad range of oxidative transformations (Ullrich et al., 2004). These aromatic peroxygenases, later renamed as unspecific peroxygenases (UPOs, E.C. 1.11.2.1), are extracellular fungal enzymes, which form one among five members of a new sub-subclass of oxidoreductases (E.C. 1.11.2.-). Unlike the related group of chloroperoxidases (CPOs, E.C. 1.11.1.10), UPOs have minor natural catalytic activity toward chlorination reactions but otherwise efficiently catalyze a broad range of selective oxyfunctionalization of non-activated $\mathrm{C}-\mathrm{H}$ - and $\mathrm{C}=\mathrm{C}$-bonds as well as C-C-bond cleavage. In contrast to cytochrome P450 monooxygenases (P450, E.C. 1.14.---), UPOs 
do not rely on electron donating flavin- or iron-sulfur-containing redox partners; instead they only require $\mathrm{H}_{2} \mathrm{O}_{2}$, which serves as both electron acceptor and oxygen donor, and the peroxygenase can thus be considered as self-sufficient (Ullrich and Hofrichter, 2005). Resting state UPOs contain the ferric heme with a loosely bound water ligand, which is then replaced by hydrogen peroxide to form the peroxo-complex Compound 0 and subsequently the key intermediate Compound I, an oxo-ferryl cation radical complex. This strong oxidant abstracts a hydrogen atom from the substrate, forming the protonated intermediate Compound II and finally releasing the hydroxylated product after rapid recombination of the short-lived substrate radical. Since UPOs can perform one-electron and two-electron oxidations, they are assumed to be the "missing link" between P450s and CPOs from a catalytic perspective (Hofrichter et al., 2015). Several patents on UPO-sequences and UPO-catalyzed reactions demonstrate their potential for industrial application ranging from hydroxylation of aliphatic hydrocarbons to deacylation of corticoids (e.g., Hofrichter et al., 2005, 2011; Pecyna et al., 2008; Landvik et al., 2013; Vind et al., 2014; Holla et al., 2015; Molina-Espeja et al., 2016b; Fernández-Fueyo et al., 2019).

Although UPOs have several advantageous properties, limiting factors have been identified that need to be overcome to render the biocatalysts suitable for industrial applications. For instance, frequently poor selectivity, limited enzyme activity and stability under process conditions as well as low substrate and co-substrate loadings are main issues to consider and solve (Bormann et al., 2015; Hobisch et al., 2020). In addition, UPO-catalyzed biotransformations require sufficient supply of the oxidant for substrate oxidation. However, the oxidizing agent $\mathrm{H}_{2} \mathrm{O}_{2}$ can inhibit the activity of peroxygenases and irreversibly inactivate the UPO, e.g., by degradation of the prosthetic heme group (Karich et al., 2016). The destructive effect on heme enzymes is typically observed when a larger excess of the oxidant is present (Valderrama et al., 2002). Therefore, the in situ generation of $\mathrm{H}_{2} \mathrm{O}_{2}$ by catalytic $\mathrm{O}_{2}$ reduction is the most common method balancing efficient peroxygenase activity and $\mathrm{H}_{2} \mathrm{O}_{2}$-induced inactivation. Next to enzymatic or chemical supply of $\mathrm{H}_{2} \mathrm{O}_{2}$, electrochemistry and photocatalysis are promising approaches, which have been developed (Lütz et al., 2004; Churakova et al., 2011; Horst et al., 2016; Ni et al., 2016; Schmitz et al., 2017). Moreover, limited solubility of the substrates due to their hydrophobicity is a challenging issue, which can be addressed, for example, by reaction engineering. In this context, several authors reviewed recent developments as well as challenges and opportunities of peroxygenase-catalyzed reactions regarding protein, reaction, and medium engineering approaches in detail (Bormann et al., 2015; Wang et al., 2017; Hobisch et al., 2020; Aranda et al., 2021). Furthermore, Sigmund and Poelarends provided an overview of the current state of enzymes with peroxygenase activity focusing on engineering strategies to improve oxyfunctionalization reactions (Sigmund and Poelarends, 2020). For a general overview of the fungal peroxygenase superfamily, Hofrichter et al. (2020) summarized the state of knowledge of basic and applied UPO research including phylogeny, protein structure, and catalytic activity.
The aim of this contribution was therefore to review the current state of the art in UPO identification, recombinant expression, and screening approaches along with a brief overview of the status of the sustainable biotechnological applicability of UPOs. In particular, the following tools for selection of putative UPOs as well as UPO synthesis and characterization are discussed: (1) genome mining approaches to identify new candidates, (2) efficient expression systems for protein production, and (3) high-throughput methods for time-saving evaluation of enzyme activity.

\section{GENOME MINING APPROACHES TO IDENTIFY NEW UPOS}

Due to their promiscuity for oxygen transfer reactions, UPOs have been focused on both in basic and applied research. According to phylogenetic analysis, UPOs can generally be divided into two families: Family I, which includes "shorttype" UPOs, and family II containing "long-type” UPOs (Kellner et al., 2014; Hofrichter et al., 2015). To date, family I comprises more than 1,100 short-type UPOs, occurring in most fungal phyla, with a mean size of around $30 \mathrm{kDa}$, but mainly without predictable signal peptides. Nonetheless, signal peptidecontaining and thus secreted UPOs such as the characterized UPOs from Chaetomium globosum (CglUPO), Marasmius rotula (MroUPO) and Marasmius wettsteinii (MweUPO) as well as well-established chloroperoxidase from Leptoxyphium (Caldariomyces) fumago ( LfuCPO, also known as CfuCPO) are assigned to this short-type family (Hofrichter et al., 2020). Both characterized Marasmius-UPOs are assumed to be dimeric proteins linked by intermolecular disulfide bridges to connect the monomers. Intramolecular bridges, however, are not present (Olmedo et al., 2017; Ullrich et al., 2018). Family II harbors more than 900 long-type UPOs along with the well-studied model enzyme AaeUPO from Agrocybe aegerita. Family II UPOs have a mean size of $44.4 \mathrm{kDa}$ and can only be found in Ascomycota and Basidiomycota. Here, most sequences possess a C-terminal disulfide bridge and predicted signal peptides, which indicates the extracellular location of the protein. Both UPO families contain highly conserved amino acid motifs essential for their catalytic functionality. The motifs -EHD-S-E- and -EGD-S-RE- are found in family I and II, respectively, along with the characteristic PCP motif in both families. On the other hand, there are several structural differences in UPO architecture, such as the heme access channel, which might explain the differences in substrate specificity (Hofrichter et al., 2020). For instance, neither MroUPO nor AaeUPO convert the sterically demanding compound testosterone, while the recently identified CglUPO is able to oxidize the steroid molecule (Kiebist et al., 2017).

Recently, Faiza et al. (2019a) searched for novel UPOs by genome mining of more than 800 fungal genomes and identified 113 putative sequences in 35 fungal species. Based on phylogenetic analysis and motif patterns, a new classification of UPOs with five subfamilies was proposed, in which AaeUPO and CciUPO from Coprinopsis cinerea belong to subfamily I, while $L f u C P O$ and $M r o U P O$ are classified in a separate 
peroxidase-peroxygenase (Pog) superfamily between classical CPOs and UPOs. Furthermore, they created the first UPO online database called "Unspecific Peroxygenase Database" (UPObase) that provides more than 1,900 putative UPO protein sequences including information about classification and motifs as well as homology search tools such as multiple sequence alignments and phylogenetic trees (Faiza et al., 2019b). However, the complex phylogenetic organization of UPO genes and the physiological background of certain amino acid residues are not yet completely understood.

At a molecular level, crystal structures of well-studied model enzymes LfuCPO (1CPO; Sundaramoorthy et al., 1995), AaeUPO (2YOR; Piontek et al., 2013), MroUPO (5FUJ; Piontek et al., 2017) as well as laboratory-evolved PaDa-I mutant (5OXU; Ramirez-Escudero et al., 2018) have been elucidated in recent years. Growing knowledge of the protein sequence of UPOs led to the identification of numerous UPO-like genes via database search in sequenced organisms. With an increasing availability of whole genome sequences and thus an increasing number of annotated UPOs, it is no longer a matter of the accessibility of gene sequences but of the correct determination of the genefunction relationship. Figure $\mathbf{1}$ demonstrates the divergence between the number of genomic sequences annotated as putative UPOs deposited in UniProtKB and the number of verified UPOs over the last two decades. In fact, NCBI currently provides more than 2,500 fungal genomes with thousands of putative UPO sequences mainly in Ascomycetes and Basidiomycetes. On the contrary, to the best of our knowledge, only nine ascomycetous and eight basidiomycetous UPOs (excluding protein isoforms) have been purified and characterized to date, resulting in more than 4,800 non-characterized peroxygenase sequences. In addition to the wild-type UPO protein, the high number of UPO variants generated by directed evolution approaches (e.g., 9,000 clones by Molina-Espeja et al., 2014) further emphasizes the characterization gap between identified or generated candidates and evaluation of their catalytic activity. Current attempts to close this gap are discussed in the following.

\section{UPO PRODUCTION APPROACHES}

Despite the great potential of UPOs for biotechnological purposes, practical application of this novel enzyme subclass harbors several technical bottlenecks, such as the often limited enzyme activity and stability along with frequently very low reactant concentrations (Bormann et al., 2015). In general, a precondition for protein engineering and enzyme application on industrial scale is their homologous or heterologous expression at high yields. Therefore, the application of UPO proteins in the pharmaceutical or fine chemical industry will only be feasible if a cost-effective and reliable biocatalyst production in the "g-per-liter" range is realized. In particular, the widespread yeast workhorse Pichia pastoris (syn. Komagataella phaffii) produced recombinant mammalian proteins with a titer of up to $20 \mathrm{~g} \mathrm{~L}^{-1}$, while filamentous fungi like Aspergillus niger and Trichoderma reese $i$ were able to secrete $25 \mathrm{~g} \mathrm{~L}^{-1}$ of glucoamylase (A. niger) and $20-30 \mathrm{~g} \mathrm{~L}^{-1}$ of cellulase (T. reesei) (Durand et al., 1988; Demain and Vaishnav, 2009). To date, UPOs have been secreted in their native fungal producers (Ullrich et al., 2004, 2018; Anh et al., 2007; Gröbe et al., 2011; Kiebist et al., 2017; Kimani, 2019) or heterologously expressed in

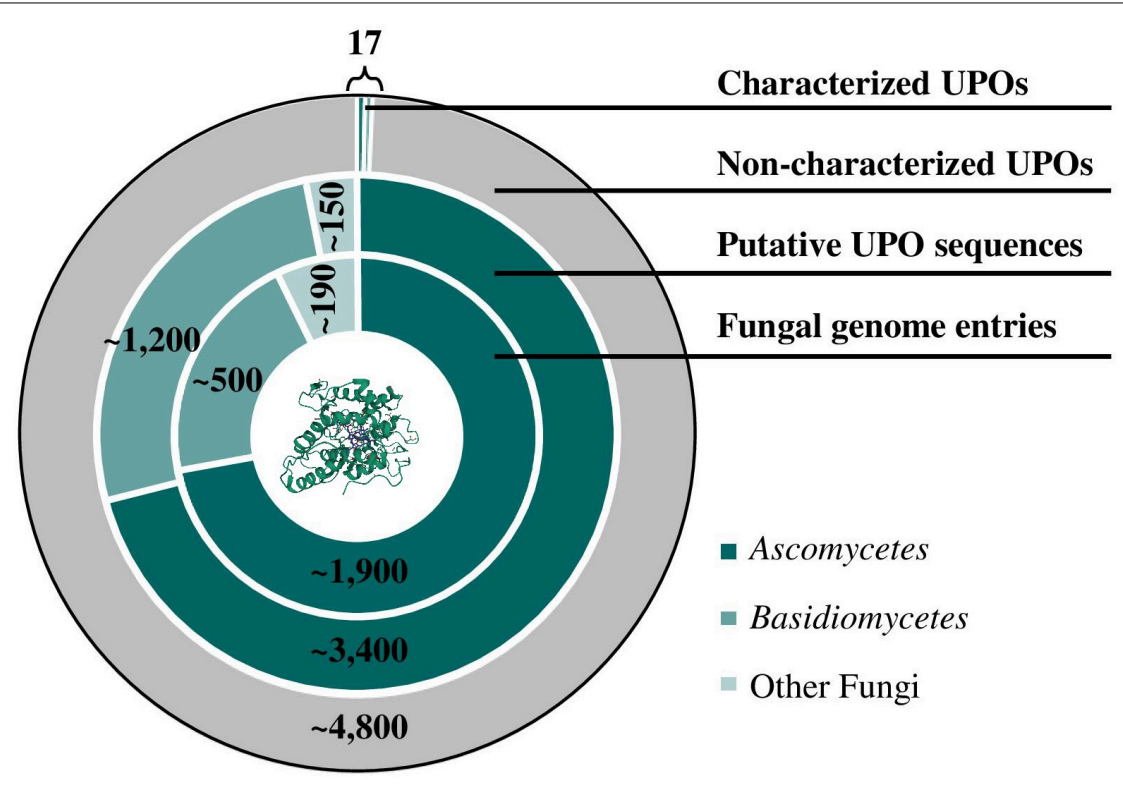

FIGURE 1 | Characterization gap of UPO proteins. Database searches for putative UPO sequences were performed by matching the conversed heme haloperoxidase family profile ("PS51405") deposited on PROSITE with protein sequences stored in the UniProtKB protein database. Nine ascomycetous and eight basidiomycetous UPOs (excluding protein isoforms) have been purified and characterized to date. Currently, approximately 2,700 fungal genome entries are listed in NCBI. (PDB ID 5OXU; Ramirez-Escudero et al., 2018). 
A. niger (Conesa et al., 2001), Aspergillus oryzae (Pecyna et al., 2008), Escherichia coli (Carro et al., 2019), P. pastoris, and Saccharomyces cerevisiae (Molina-Espeja et al., 2014, 2015) at the "mg-per-liter" level. Major challenges for efficient UPO synthesis are certainly the signal peptide (e.g., insufficient interaction between signal recognition particle (SRP) and hydrophobic region of the signal peptide), post-translational modifications (e.g., high degree of glycosylation) and the lack of chaperones in especially heterologous host organisms. Interestingly, natural hyperglycosylation by $S$. cerevisiae appeared to have a beneficial effect on enzyme activity of evolved AaeUPO mutants, probably due to increased stability, as well as optimization of the 43-aminoacid signal peptide led to markedly enhanced protein secretion (Molina-Espeja et al., 2014). When expressed in prokaryotic E. coli, codon optimization of the fungal gene sequence combined with reduced induction using an auto-induction growth medium and a low incubation temperature $\left(16^{\circ} \mathrm{C}\right)$ resulted in correctly folded active UPO proteins (Linde et al., 2020).

Table 1 provides a detailed overview of current UPO expression and purification approaches including native and recombinant producers. In addition to the already characterized UPO proteins, UPO activity has been detected without further purification in the fungal species Agaricus bisporus, Agrocybe alnetorum, Agrocybe chaxingu, Agrocybe parasitica, Auricularia auricula-judae, and Mycena galopus (Hofrichter et al., 2015). In vitro UPO expression approaches using cellfree protein synthesis technologies have not yet been reported but harbor high potential for high-throughput enzyme screening (Rolf et al., 2019).

\section{Using the Natural Host}

Currently, eight enzymes were produced by their respective natural host (see Table 1). Fungal UPO production is routinely conducted in carbon- and nitrogen-rich plant-based media in agitated flasks or stirred-tank bioreactors holding a vessel volume from $100 \mathrm{~mL}$ to $5 \mathrm{~L}$ (Ullrich et al., 2004; Anh et al., 2007; Gröbe et al., 2011). Therefore, the production of PabUPO from basidiomycetous fungus Psathyrella aberdarensis in $30 \mathrm{~L}$ scale appears to be an outlier upward among expression approaches using native producers (Kimani, 2019). As frequently observed, medium composition has a crucial influence on the UPO production success (Hofrichter et al., 2015). For instance, UPOmodel fungus $A$. aegerita TM A1 significantly varied in UPO expression level when amounts of soybean meal and Bacto Peptone were changed or even when the same fermentation conditions were repeated (Ullrich et al., 2004, 2009). Therefore, for each fungal strain used for UPO production, growth medium and cultivation conditions must be optimized in advance.

Laborious genetic engineering, like construction and transformation of recombinant DNA, is not necessary for the production of unmodified, wild-type UPO proteins with their respective wild-type fungus. Unfortunately, further protein optimization is not possible due to lacking genetic engineering tools for these species. In general, however, the production process is time-consuming because UPO secretion only starts during secondary metabolism phase and reaches its maximum after 2-4 weeks of cultivation. Probably due to non-homogenous growth of most filamentous fungi, biomass was not quantified in the majority of fermentation approaches. Alternatively, maximum enzyme activity serves as an indicator for cultivation termination and harvest, which occurred between 10 and 24 days of fermentation (see Table 1).

As UPOs are extracellular enzymes, protein quantification and activity analysis are possible using the culture supernatant without further cell disruption. Nevertheless, detailed enzyme characterization requires UPO protein purification, which is typically performed in several steps comprising ultrafiltration, multistep fast protein liquid chromatography (FPLC) using anion and cation exchangers as well as size exclusion columns, based on the published method of Hofrichter and colleagues in 2004 (Ullrich et al., 2004). In general, UPO production and subsequent purification appear to be an issue, as the recovery yield of purified enzymes averages $10 \%$ combined with low amounts of total protein $(\sim 1 \%$ protein yield). So far, the highest yield was achieved with MweUPO expressed by the saprotrophic basidiomycete $M$. wettsteinii resulting in $26 \%$ recovery yield after four FPLC steps (Ullrich et al., 2018). In comparison, five steps were required for purification of MroUPO from related fungus $M$. rotula with an about 40-times lower final yield of $0.7 \%$ (Gröbe et al., 2011). However, LfuCPO from fastgrowing ascomycete L. fumago seems to be an outlier among the other UPO proteins with a recovery yield of $70.4 \%$ and a protein yield of $\sim 24 \%$. Unlike most UPOs, $L f u C P O$ production is routinely conducted in defined fructose-salt medium, while purification is based on aqueous biphasic extraction followed by dialysis and anion exchange chromatography (Pickard, 1981; Yazbik and Ansorge-Schumacher, 2010). In fact, medium and purification optimization were focused to reduce contamination by black pigmentation accompanying cultivation. Efficient and fast purification methods led to the remarkable enzyme recovery with high activity of the chloroperoxidase (purified LfuCPO $2,900 \mathrm{U} \mathrm{mg}^{-1}$ toward halogenation of monochlorodimedone). In a different approach, elimination of black pigmentation was addressed by mutagenesis using UV irradiation and resulted in L. fumago mutant strain white2 with up to 2.3-fold higher specific productivity and earlier CPO secretion (Buchhaupt et al., 2012). However, to our knowledge, no comparable experiments with other UPO enzymes have been published yet.

In contrast to the widely used model organisms E. coli, $S$. cerevisiae and $P$. pastoris, genetic accessibility of fungal UPOsecreting strains is low and only a few molecular tools for $A$. aegerita and $C$. cinerea are available up to now (Sugano et al., 2017; Herzog et al., 2019). In this context, LfuCPO is the only UPO protein expressed by homologous over-expression in the recombinant L. fumago strain Cf-CPO4. A CPO-expression cassette was integrated into non-transcribed spacer regions of the ribosomal DNA in L. fumago resulting an increase in protein concentration to $\sim 1.95 \mathrm{~g} \mathrm{~L}^{-1}$ compared to $\sim 1.2 \mathrm{~g} \mathrm{~L}^{-1}$ in culture liquid of wild-type L. fumago (Buchhaupt et al., 2011). Unfortunately, homologous over-expression of $L f u C P O$ was not subject of further published work at this stage. In general, limited or no data on homologous over-expression of UPOs have been available. Therefore, heterologous expression, which allows the biosynthesis of an enzyme from an organism other than its 
TABLE 1 | Current UPO expression approaches using native and heterologous producer organisms.

\begin{tabular}{|c|c|c|c|c|c|c|c|c|c|c|c|}
\hline \multirow[b]{2}{*}{ Enyzme } & \multirow[b]{2}{*}{ Expression organism } & \multirow[b]{2}{*}{ Culture volume [L] } & \multirow[b]{2}{*}{$\begin{array}{c}\text { Cultivation } \\
\text { duration }\end{array}$} & \multicolumn{2}{|c|}{ Culture liquid } & \multicolumn{5}{|c|}{ Purified enzyme } & \multirow[b]{2}{*}{ References } \\
\hline & & & & $\begin{array}{c}\text { Total } \\
\text { protein } \\
{\left[\mathrm{mg} \mathrm{L}^{-1}\right]}\end{array}$ & $\begin{array}{l}\text { Specific } \\
\text { activity } \\
{\left[\mathrm{Ug}^{-1}\right]}\end{array}$ & $\begin{array}{c}\text { Total } \\
\text { protein } \\
{\left[\mathrm{mg} \mathrm{L}^{-1}\right]}\end{array}$ & $\begin{array}{c}\text { Specific } \\
\text { activity } \\
{\left[\mathrm{U} \mathrm{mg}^{-1}\right]}\end{array}$ & $\begin{array}{l}\text { Recovery } \\
\text { yield [\%] }\end{array}$ & $\begin{array}{l}\text { Protein } \\
\text { yield [\%] }]^{b}\end{array}$ & $\begin{array}{l}\text { Purification step } \\
\text { (purification } \\
\text { factor) }\end{array}$ & \\
\hline \multicolumn{12}{|c|}{ Protein expression in native producers } \\
\hline AaeUPO & Agrocybe aegerita & 3.9 (BR) & 11 days & $119.23^{a}$ & $3^{\mathrm{e}}$ & $0.38^{\mathrm{a}}$ & $165^{\mathrm{e}}$ & 18 & 0.32 & IEX (55) & Ullrich et al., 2004 \\
\hline Cg/UPO & Chaetomium globosum & $0.2(\mathrm{SF})$ & 21 days & $\sim 40$ & $0.6^{\mathrm{e}}$ & $120^{\mathrm{a}}$ & $12^{\mathrm{e}}$ & 8.5 & 0.42 & IEX (20) & Kiebist et al., 2017 \\
\hline CraUPO & Coprinellus radians & 0.2 (total 4 L f.p.) & 13 days & $188.10^{\mathrm{a}}$ & $0.83^{e}$ & $0.14^{\mathrm{a}}$ & $38.5^{\mathrm{e}}$ & 3.3 & 0.07 & SEC (46.2) & Anh et al., 2007 \\
\hline CreuPO & Coprinus verticillatus & 0.04 (SF) & 21 days & n.a. & n.a. & n.p. & n.p. & n.p. & n.p. & n.p. & $\begin{array}{l}\text { Anh et al., } 2007 \text { (later } \\
\text { purified according to } \\
\text { Hofrichter et al., 2020) }\end{array}$ \\
\hline \multirow[t]{4}{*}{ LfuCPO } & Leptoxyphium fumago & 0.4 (SF) & 10-12 days & n.a. & $1001.6^{f}$ & n.a. & $2,900.10^{f}$ & 70.4 & 24.27 & $\operatorname{IEX}(2.9)$ & $\begin{array}{l}\text { Yazbik and } \\
\text { Ansorge-Schumacher, } \\
2010\end{array}$ \\
\hline & Leptoxyphium fumago & $1.02(\mathrm{BR})$ & 13 days & $\sim 1,206^{g}$ & n.a. & n.p. & n.p. & n.p. & n.p. & n.p. & Buchhaupt et al., 2011 \\
\hline & L. fumago Cf-CPO4 & $1.02(\mathrm{BR})$ & 13 days & $\sim 1,950$ & n.a. & n.p. & n.p. & n.p. & n.p. & n.p. & Buchhaupt et al., 2011 \\
\hline & Marasmius rotula & 0.2 (SF) & 28 days & 445 & n.a. & n.p. & n.p. & n.p. & n.p. & n.p. & Gröbe et al., 2011 \\
\hline MroUPO & Marasmius rotula & 4 (0.468 L f.p.) & 24 days & 280 & $16^{e}$ & $2.09^{a}$ & $76^{e}$ & 0.7 & 0.13 & IEX (4.8) & Gröbe et al., 2011 \\
\hline MweUPO & Marasmius wettsteinii & 0.2 (SF) & 21 days & $11,320^{\mathrm{a}, \mathrm{c}}$ & $1.6^{\mathrm{e}}$ & $120^{\mathrm{a}}$ & $37.5^{\mathrm{e}}$ & 26 & 1.06 & IEX (24) & Ullrich et al., 2018 \\
\hline PabUPO & Psathyrella aberdarensis & 15 (BR) & 17 days & $77.40^{\mathrm{a}}$ & $2.4^{\mathrm{e}}$ & $0.20^{a}$ & $73.7^{e}$ & 7 & 0.26 & IEX (30.8) & Kimani, 2019 \\
\hline \multicolumn{12}{|c|}{ Heterologous protein expression } \\
\hline \multirow[t]{2}{*}{ rAaeUPO } & Saccharomyces cerevisiae & 1 & $72 \mathrm{~h}$ & $\sim 0.007$ & n.a. & n.p. & n.p. & n.p. & n.p. & n.p. & $\begin{array}{l}\text { Molina-Espeja et al., } \\
2014\end{array}$ \\
\hline & Pichia pastoris & n.a. & n.a. & n.d. & n.a. & n.a. & n.a. & n.a. & n.a. & n.a. & $\begin{array}{l}\text { Molina-Espeja et al., } \\
2015\end{array}$ \\
\hline \multirow[t]{2}{*}{ rCg/UPO } & Saccharomyces cerevisiae & 0.5 (SF; total 1 L f.p.) & $72 \mathrm{~h}$ & n.a. & n.a. & 0.6 & n.a. & n.a. & n.a. & $A C$ & Püllmann et al., 2021 \\
\hline & Pichia pastoris & 0.5 (SF; total 1 L f.p.) & $72 \mathrm{~h}$ & n.a. & n.a. & 9 & n.a. & n.a. & n.a. & $A C$ & Püllmann et al., 2021 \\
\hline rGmaUPO & Saccharomyces cerevisiae & 0.5 (SF; total 1 L f.p.) & $72 \mathrm{~h}$ & n.a. & n.a. & n.a. & n.a. & n.a. & n.a. & n.a. & Püllmann et al., 2021 \\
\hline rMfeUPO & Pichia pastoris & 0.5 (SF; total 1 L f.p.) & $72 \mathrm{~h}$ & n.a. & n.a. & 6.5 & n.a. & n.a. & n.a. & $\mathrm{AC}$ & $\begin{array}{l}\text { Püllmann and } \\
\text { Weissenborn, } 2021\end{array}$ \\
\hline rMhiUPO & Pichia pastoris & 0.5 (SF; total 1 L f.p.) & $72 \mathrm{~h}$ & n.a. & n.a. & 5.7 & n.a. & n.a. & n.a. & $A C$ & $\begin{array}{l}\text { Püllmann and } \\
\text { Weissenborn, } 2021\end{array}$ \\
\hline \multirow[t]{2}{*}{ rMthUPO } & Saccharomyces cerevisiae & 0.5 (SF; total 1 L f.p.) & $72 \mathrm{~h}$ & n.a. & n.a. & 5 & n.a. & n.a. & n.a. & $A C$ & Püllmann et al., 2021 \\
\hline & Pichia pastoris & 0.5 (SF; total 1 L f.p.) & $72 \mathrm{~h}$ & n.a. & n.a. & 24 & n.a. & n.a. & n.a. & $A C$ & Püllmann et al., 2021 \\
\hline rMweUPO & Saccharomyces cerevisiae & 0.5 (SF; total 1 L f.p.) & $72 \mathrm{~h}$ & n.a. & n.a. & n.a. & n.a. & n.a. & n.a. & n.a. & Püllmann et al., 2021 \\
\hline $\begin{array}{l}\text { PaDa-I } \\
\text { (rAaeUPO } \\
\text { variant) }\end{array}$ & Saccharomyces cerevisiae & 1 & $72 \mathrm{~h}$ & 0.14 & $25^{d}$ & 0.21 & $828^{d}$ & n.a. & n.a. & IEX (33.1) & $\begin{array}{l}\text { Molina-Espeja et al., } \\
2014\end{array}$ \\
\hline
\end{tabular}


TABLE 1 | Continued

\begin{tabular}{|c|c|c|c|c|c|c|c|c|c|c|c|}
\hline \multirow[b]{2}{*}{ Enyzme } & \multirow[b]{2}{*}{ Expression organism } & \multirow[b]{2}{*}{ Culture volume [L] } & \multirow[b]{2}{*}{$\begin{array}{c}\text { Cultivation } \\
\text { duration }\end{array}$} & \multicolumn{2}{|c|}{ Culture liquid } & \multicolumn{5}{|c|}{ Purified enzyme } & \multirow[b]{2}{*}{ References } \\
\hline & & & & $\begin{array}{c}\text { Total } \\
\text { protein } \\
{\left[\mathrm{mg} \mathrm{L}^{-1}\right]}\end{array}$ & $\begin{array}{l}\text { Specific } \\
\text { activity } \\
{\left[\mathrm{U} \mathrm{mg}^{-1}\right]}\end{array}$ & $\begin{array}{c}\text { Total } \\
\text { protein } \\
{\left[\mathrm{mg} \mathrm{L}^{-1}\right]}\end{array}$ & $\begin{array}{l}\text { Specific } \\
\text { activity } \\
{\left[\mathrm{U} \mathrm{mg}^{-1}\right]}\end{array}$ & $\begin{array}{l}\text { Recovery } \\
\text { yield [\%] }\end{array}$ & $\begin{array}{c}\text { Protein } \\
\text { yield }[\%]^{b}\end{array}$ & $\begin{array}{l}\text { Purification step } \\
\text { (purification } \\
\text { factor) }\end{array}$ & \\
\hline \multirow{3}{*}{$\begin{array}{l}\text { PaDa-I } \\
\text { (rAaeUPO } \\
\text { variant) }\end{array}$} & Saccharomyces cerevisiae & 0.275 (BR; fed-batch) & 6 days & n.a. & n.a. & n.a. & n.a. & n.a. & n.a. & n.a. & $\begin{array}{l}\text { Molina-Espeja et al., } \\
2015\end{array}$ \\
\hline & Pichia pastoris & 4.4 (BR; fed-batch) & 6 days & 217 & n.a. & $\sim 8$ & n.a. & n.a. & n.a. & n.a. & $\begin{array}{l}\text { Molina-Espeja et al., } \\
2015\end{array}$ \\
\hline & Pichia pastoris & 0.5 (SF; total 1 L f.p.) & $72 \mathrm{~h}$ & n.a. & n.a. & 12.6 & n.a. & n.a. & n.a. & $\mathrm{AC}$ & $\begin{array}{l}\text { Püllmann and } \\
\text { Weissenborn, } 2021\end{array}$ \\
\hline rCciuPO & Aspergillus oryzae & n.a. & n.a. & n.a. & n.a. & n.a. & $100^{e}$ & n.a. & n.a. & n.a. & Babot et al., 2013 \\
\hline \multirow[t]{2}{*}{ rCviuPO } & Escherichia coli & 3 & $4-5$ days & n.a. & n.a. & n.a. & $38.2^{d}$ & 25 & n.a. & n.a. & $\begin{array}{l}\text { González-Benjumea } \\
\text { et al., } 2020\end{array}$ \\
\hline & Escherichia coli & 8 & $4-5$ days & $831.25^{\mathrm{a}}$ & $\begin{array}{l}0.17^{d} \\
0.01^{e}\end{array}$ & 7 & $38.18^{\mathrm{d}}, 1.7^{\mathrm{e}}$ & 27 & 0.12 & Ultrafiltration (25) & Linde et al., 2020 \\
\hline \multirow[t]{2}{*}{ rDcaUPO } & Escherichia coli & 10 & $4-5$ days & $423^{a}$ & $\begin{array}{l}0.06^{d} \\
0.01^{e}\end{array}$ & 2.8 & $7.68^{\mathrm{d}}, 1.62^{\mathrm{e}}$ & 13 & 0.09 & Ultrafiltration (103) & Linde et al., 2020 \\
\hline & Pichia pastoris & 0.5 (SF; total 1 L f.p.) & $72 \mathrm{~h}$ & n.a. & n.a. & 16.3 & n.a. & n.a. & n.a. & $\mathrm{HIC}$ & $\begin{array}{l}\text { Püllmann and } \\
\text { Weissenborn, } 2021\end{array}$ \\
\hline rHinUPO & Aspergillus oryzae & n.a. & n.a. & n.a. & n.a. & n.a. & $5.4^{e}$ & n.a. & n.a. & n.a. & Peter et al., 2014 \\
\hline rLfuCPO & Aspergillus niger & 0.5 (SF; total 1 L f.p.) & $48 \mathrm{~h}$ & 10 & n.a. & n.a. & $39,000 \cup L^{-1 f}$ & 11 & n.a. & GPC & Conesa et al., 2001 \\
\hline \multirow[t]{3}{*}{ rMroUPO } & Escherichia coli & n.a. & 4 days & n.a. & n.a. & n.a. & n.a. & n.a. & n.a. & n.a. & Carro et al., 2019 \\
\hline & Saccharomyces cerevisiae & 0.5 (SF; total 1 L f.p.) & $72 \mathrm{~h}$ & n.a. & n.a. & 0.35 & n.a. & n.a. & n.a. & $A C$ & Püllmann et al., 2021 \\
\hline & Pichia pastoris & 0.5 (SF; total 1 L f.p.) & $72 \mathrm{~h}$ & n.a. & n.a. & 1.1 & n.a. & n.a. & n.a. & $\mathrm{AC}$ & Püllmann et al., 2021 \\
\hline \multirow[t]{2}{*}{ rTteUPO } & Saccharomyces cerevisiae & 0.5 (SF; total 1 L f.p.) & $72 \mathrm{~h}$ & n.a. & n.a. & 17 & n.a. & n.a. & n.a. & $\mathrm{AC}$ & Püllmann et al., 2021 \\
\hline & Pichia pastoris & 0.5 (SF; total 1 L f.p.) & $72 \mathrm{~h}$ & n.a. & n.a. & 21.9 & n.a. & n.a. & n.a. & $A C$ & $\begin{array}{l}\text { Püllmann and } \\
\text { Weissenborn, } 2021\end{array}$ \\
\hline
\end{tabular}

${ }^{a}$ Calculated from culture volume.

${ }^{b}$ Calculated from amount of total protein of culture liquid and purified enzyme.

c Ultrafiltrate.

${ }^{d}$ ABTS assay.

e Veratryl alcohol assay.

${ }^{f}$ Monochlorodimedone assay.

${ }^{g}$ Calculated according to Buchhaupt et al., 2011.

AC, affinity chromatography; BR, bioreactor; f.p., for purification; GPC, gel permeation chromatography; HIC, hydrophobic interaction chromatography; IEX, ion exchange chromatography; n.a., not available; n.d., not detectable; n.p., not purified; SEC, size exclusion chromatography; SF, shake flask. 
TABLE 2 | High-throughput screening approaches for UPO mutant library analysis.

\begin{tabular}{|c|c|c|c|c|c|}
\hline $\begin{array}{l}\text { Directed evolution mutagenesis } \\
\text { method }\end{array}$ & Parental enzyme & $\begin{array}{l}\text { Expression } \\
\text { host }\end{array}$ & $\begin{array}{l}\text { Screened } \\
\text { clones }\end{array}$ & Assay method & References \\
\hline $\begin{array}{l}\text { Error-prone PCR; in vivo DNA shuffling; } \\
\text { in vivo assembly of mutant libraries; } \\
\text { MORPHING; site-directed mutagenesis }\end{array}$ & rAaeUPO & S. cerevisiae & $\sim 9,000$ & ABTS; NBD & Molina-Espeja et al., 2014 \\
\hline Error-prone PCR; in vivo DNA shuffling & $\begin{array}{l}\text { Evolved rAaeUPO } \\
\text { PaDa-I }\end{array}$ & S. cerevisiae & $\sim 7,000$ & ABTS & Martin-Diaz et al., 2018 \\
\hline MORPHING; saturation mutagenesis & $\begin{array}{l}\text { Evolved rAaeUPO } \\
\text { PaDa-I }\end{array}$ & S. cerevisiae & $\sim 4,500$ & ABTS; NBD & Mate et al., 2017 \\
\hline $\begin{array}{l}\text { Error-prone PCR; staggered extension } \\
\text { process recombination combined with } \\
\text { in vivo shuffling }\end{array}$ & $\begin{array}{l}\text { Evolved rAaeUPO } \\
\text { PaDa-I }\end{array}$ & S. cerevisiae & $\sim 4,000$ & $\begin{array}{l}\text { Naphthalene-Fast Red; } \\
\text { DMP }\end{array}$ & Molina-Espeja et al., 2016a \\
\hline $\begin{array}{l}\text { Combined promoter and signal peptide } \\
\text { shuffling system }\end{array}$ & $\begin{array}{l}\text { rAaeUPO; rDcaUPO; } \\
\text { rMfeUPO; rMhiUPO; } \\
\text { rMthUPO; rTteUPO }\end{array}$ & P. pastoris & $\sim 3,200$ & NBD; DMP & $\begin{array}{l}\text { Püllmann and } \\
\text { Weissenborn, 2021; } \\
\text { Püllmann et al., } 2021\end{array}$ \\
\hline MORPHING; saturation mutagenesis & $\begin{array}{l}\text { Evolved rAaeUPO } \\
\text { JaWa }\end{array}$ & S. cerevisiae & $\sim 3,000$ & 4-aminoantypirine (4-AAP) & $\begin{array}{l}\text { Gómez de Santos et al., } \\
2018\end{array}$ \\
\hline $\begin{array}{l}\text { Golden Mutagenesis (Püllmann et al., } \\
\text { 2019) }\end{array}$ & rMthUPO & S. cerevisiae & $\sim 900$ & $\begin{array}{l}\text { Octane; cyclohexane; } \\
\text { cyclohexene } \\
\text { (MISER-GC-MS) }\end{array}$ & Knorrscheidt et al., 2021 \\
\hline Shuffled peroxygenase gene library & $\begin{array}{l}\text { PaDa-I; rGmaUPO; } \\
\text { rCciUPO }\end{array}$ & S. cerevisiae & $\begin{array}{l}672 \text { (screened } \\
\text { in } 7 \mathrm{~h} \text { ) }\end{array}$ & $\begin{array}{l}\text { 1,2,3,4- } \\
\text { tetrahydronaphthalene } \\
\text { (MISER-GC-MS) }\end{array}$ & $\begin{array}{l}\text { Knorrscheidt et al., 2019, } \\
2020\end{array}$ \\
\hline $\begin{array}{l}\text { MORPHING; in vivo assembly of mutant } \\
\text { libraries; site-directed mutagenesis }\end{array}$ & rAaeUPO & S. cerevisiae & $\begin{array}{l}\text { 500 per } \\
\text { library }\end{array}$ & ABTS; NBD & Gonzalez-Perez et al., 2014 \\
\hline
\end{tabular}

MORPHING, mutagenic organized recombination process by homologous in vivo grouping; MISER, multiple injections in a single experimental run.

natural producer, has been targeted in several approaches over the last decade.

\section{Using Heterologous Expression}

Since genetical modification of filamentous fungi involves a number of challenges and different transformation techniques are required for different fungal species (Li et al., 2017; Lichius et al., 2020), the current focus is on heterologous protein expression in model organisms to bring versatile UPOs closer to large-scale use and industrial application. First successful attempts of heterologous UPO expression were published almost a decade after the first UPO characterization in 2013 using the mold $A$. oryzae followed by the yeast organism S. cerevisiae in 2014 (Babot et al., 2013; Molina-Espeja et al., 2014). Very recently, soluble UPO expression in prokaryotic $E$. coli cells as active enzyme has been reported and further disclosed by an international patent (Fernández-Fueyo et al., 2019), since former efforts of UPO over-expression as bacterial inclusion bodies followed by in vitro activation were unsuccessful (Carro et al., 2019; Linde et al., 2020). Excluding the previously conducted genetic engineering work, duration of protein production by heterologous expression could be reduced to $72 \mathrm{~h}$ of cultivation using yeast as well as 4-5 days using E. coli (see Table 1). In addition, the purification of UPO enzymes can be simplified by using tagged proteins and affinity chromatography. However, recombinant production of wild-type UPOs, without further optimization of the signal peptide, usually varies depending on the protein. For instance, rAaeUPO expressed in yeast resulted in almost undetectable amounts of protein (Molina-Espeja et al., $2014,2015)$, while $\mathrm{r} L f u C P O$ was produced in $A$. niger with a titer of $\sim 10 \mathrm{mg} \mathrm{L}^{-1}$ and shake flask production of $\mathrm{rCglUPO}$ and rMroUPO in $P$. pastoris yielded 9 and $1.1 \mathrm{mg} \mathrm{L}^{-1}$ enzyme, respectively (Conesa et al., 2001; Püllmann et al., 2021).

Generally, E. coli and S. cerevisiae are the most popular host organisms for directed evolution mutagenesis methods due to their versatile molecular tools available and their advantageous basis for genetic manipulation (Pourmir and Johannes, 2012). Site-directed mutagenesis was effectively used to alter and improve catalytic activity toward substrates like fatty acids by mutation of neighboring residues at the heme channel in recent approaches. Here, E. coli served as expression host for evolved rMroUPO and rCviUPO variants (Carro et al., 2019; González-Benjumea et al., 2020; Linde et al., 2020). In addition, two wild-type UPOs from the ascomycetes Collariella virescens (syn., Chaetomium virescens) and Daldinia caldariorum were successfully expressed in E. coli $\left(2.8-7 \mathrm{mg} \mathrm{L}^{-1}\right.$ in culture), purified, and catalytically characterized (Linde et al., 2020). Furthermore, for characterization and modulation of the topography of the active site in UPO enzymes, site-directed mutagenesis and subsequent expression in S. cerevisiae revealed the importance of heme channel residues, such as amino acid positions 76, 191 and 241, for substrate accessibility and catalytic activity (Gómez de Santos et al., 2019; Ramirez-Ramirez et al., 2020). In particular, substitutions F191S and G241D in an evolved rAaeUPO variant enhanced hydroxylation efficiency toward propranolol (Gómez de Santos et al., 2018), while replacement of F76 by a small residue like alanine had a negative effect on substrate affinity (Ramirez-Ramirez et al., 2020).

Due to inefficient expression capabilities of some UPOs, the optimization of wild-type peroxygenases by directed evolution 
in yeast has also been pursued in various approaches. Table 2 provides an overview of current mutagenesis attempts in yeast in combination with subsequent high-throughput screening. In general, heterologous expression of UPOs is strongly influenced by their signal peptide, as has been demonstrated during directed evolution studies for improved production of $\mathrm{rAaeUPO}$ in $S$. cerevisiae by introduction of four advantageous mutations (F[12]Y-A[14]V-R[15]G-A[21]D) that seemed to be beneficial for SRP binding and subsequent processing of the UPO polypeptide (Molina-Espeja et al., 2014). Directed evolution approaches using random as well as recombination-based mutagenesis methods in $S$. cerevisiae led to the three characterized $\mathrm{rAaeUPO}$ variants PaDa-I (Molina-Espeja et al., 2014), JaWa (based on PaDaI; Molina-Espeja et al., 2016a), and SoLo (based on JaWa; Gómez de Santos et al., 2018) with SoLo showing improved peroxygenase activity toward propranolol and lower peroxidative activity toward $5^{\prime}$-hydroxypropranolol than the parental enzyme. In a recent approach, Püllmann et al. successfully produced the new wild-type enzymes rMfeUPO from Myceliophthora fergusii, rMhiUPO from Myceliophthora hinnulea, rMthUPO from Myceliophthora thermophila and rTteUPO from Thielavia terrestris in $P$. pastoris with production titers between 5.7 and $24 \mathrm{mg} \mathrm{L}^{-1}$ after expression optimization using an episomal promoter and signal peptide shuffling system in $S$. cerevisiae (rMthUPO; rTteUPO) or P. pastoris (rMfeUPO; rMhiUPO) (Püllmann and Weissenborn, 2021; Püllmann et al., 2021). Currently, the highest titer of recombinant UPO was reported for the PaDa-I variant expressed in P. pastoris with $217 \mathrm{mg} \mathrm{L}^{-1}$ in fed-batch fermentation, while cultivation of the same $P$. pastoris strain in shake flask yielded only $\sim 8 \mathrm{mg} \mathrm{L}^{-1}$ due to lower cell densities (Molina-Espeja et al., 2015). A successful approach in the "g-per-liter" direction used the ascomycetous mold A. oryzae as fungal host to produce $\mathrm{rCciUPO}$ from Coprinopsis cinerea (Hofrichter et al., 2020). Among others, Novozymes A/S (Denmark) heterologously expresses rCciUPO as well as rHinUPO (rNOVO) from Humicola insolens at a scale of 0.2 to 15,000 L, protected by an international patent (Pecyna et al., 2008; Babot et al., 2013; Peter et al., 2014). Growing interest in fungal peroxygenases due to their catalytic potential will likely lead to optimized expression processes toward the "g-per-liter" level, e.g., by strain and reaction engineering, in the future. Consequently, this could also increase the availability of these enzymes, as so far only LfuCPO and evolved AaeUPO mutant libraries marketed by EvoEnzyme are commercially available.

\section{CURRENT UPO SCREENING STRATEGIES AND ANALYTICAL METHODS}

Screening of large enzyme libraries, which consist of protein variants or are derived from natural gene diversity, requires efficient tools applicable in biological matrices. The majority of screening assays rely on changes in absorption or fluorescence during conversion of a model substrate or on indirect sensor systems via coupled enzyme cascade reactions. These assays are mostly substrate or enzyme specific. The substances used, however, often differ from the actual substrate of interest (Kazlauskas, 2006). Therefore, more specific and comprehensive analytics are implemented such as high-performance liquid chromatography (HPLC), gas chromatography (GC) or mass spectrometry (MS) to overcome this limitation. Both approaches entail advantages and disadvantages. Colorimetric or spectrophotometric assays are simple and applicable in every lab. These assays rely on a wavelength shift during the reaction progress, based either on the used model substrate or of indirect measurements of a coupled enzyme cascade reaction or $\mathrm{H}_{2} \mathrm{O}_{2}$ depletion (Johannes et al., 2006; Reymond et al., 2009). In contrast, chromatographic assays are time-consuming and require specialized equipment but allow measurements beyond model substrates (Geueke and Kohler, 2010). Especially spectrophotometric assays were used to date to identify novel UPOs or to describe the reaction kinetics. Tables 3, 4 give an overview about the substrates and products used for colorimetric and spectrophotometric assays. In addition to these reactions, UPOs naturally catalyze a whole range of oxyfunctionalization reactions. More than 400 substrates are now known to be converted by UPOs (Hobisch et al., 2020). These reactions range from aromatic oxidations (Ramirez-Ramirez et al., 2020), epoxidation of aromatics (Zhang et al., 2021), oxidation of alcohols (Kiebist et al., 2017), dealkylation (Püllmann and Weissenborn, 2021), to oxygenation of unsaturated fatty acids (Linde et al., 2020) and halogenations (Ullrich et al., 2004).

The oxidation of 2,2'-azino-bis-(3-ethylbenzothiazoline6-sulfonic acid) (ABTS), 2,6-dimethoxyphenol (DMP), naphthalene, veratryl alcohol, and 5-nitro-1,3-benzodioxole (NBD) are the typical reactions for screening UPOs peroxidase or peroxygenase activity as they are spectrophotometricdetectable reactions (Figure 2). With the substrates ABTS and DMP, the first peroxidase activity has been proven for A. aegerita in 2004 (Ullrich et al., 2004). Moreover, DMP was used for further screenings for peroxygenase-like reactions (Molina-Espeja et al., 2016a; Breslmayr et al., 2018). Another assay was reported using NBD as substrate for peroxygenative activity screening. The product 4-nitrocatechol can be quantified at $425 \mathrm{~nm}$ over time and turns red after $\mathrm{pH}$ shift from 7 to 12 , which can be used for end-point determination at $514 \mathrm{~nm}$ (Poraj-Kobielska et al., 2012). NBD and ABTS were used in a dual-colorimetric assay to screen five generations of directed evolution comprising more than 9,000 UPO clones (Molina-Espeja et al., 2014). Activity screening with ABTS turned out to be more reliable and stable with a low interference of the culture broth and was therefore used for the first screening round. Interfering background absorption was mainly caused by the presence of hemoglobin. During directed evolution rounds, the screening substrate was changed to $\mathrm{NBD}$, when sufficient amounts of UPOs were secreted. The combination of several screening substrates makes sense in order to circumvent the respective limitations of the used substrate such as sensitivity, specificity or interference with medium compounds. Additionally, the specificity of the substrates leads to application in screenings for either peroxidative activity with ABTS or peroxygenative activity with NBD and the ratio of peroxidative:peroxygenative activity (Mate et al., 2017). A structure-guided evolution of UPOs from 
TABLE 3 | Overview of spectrophotometry-based UPO assays.

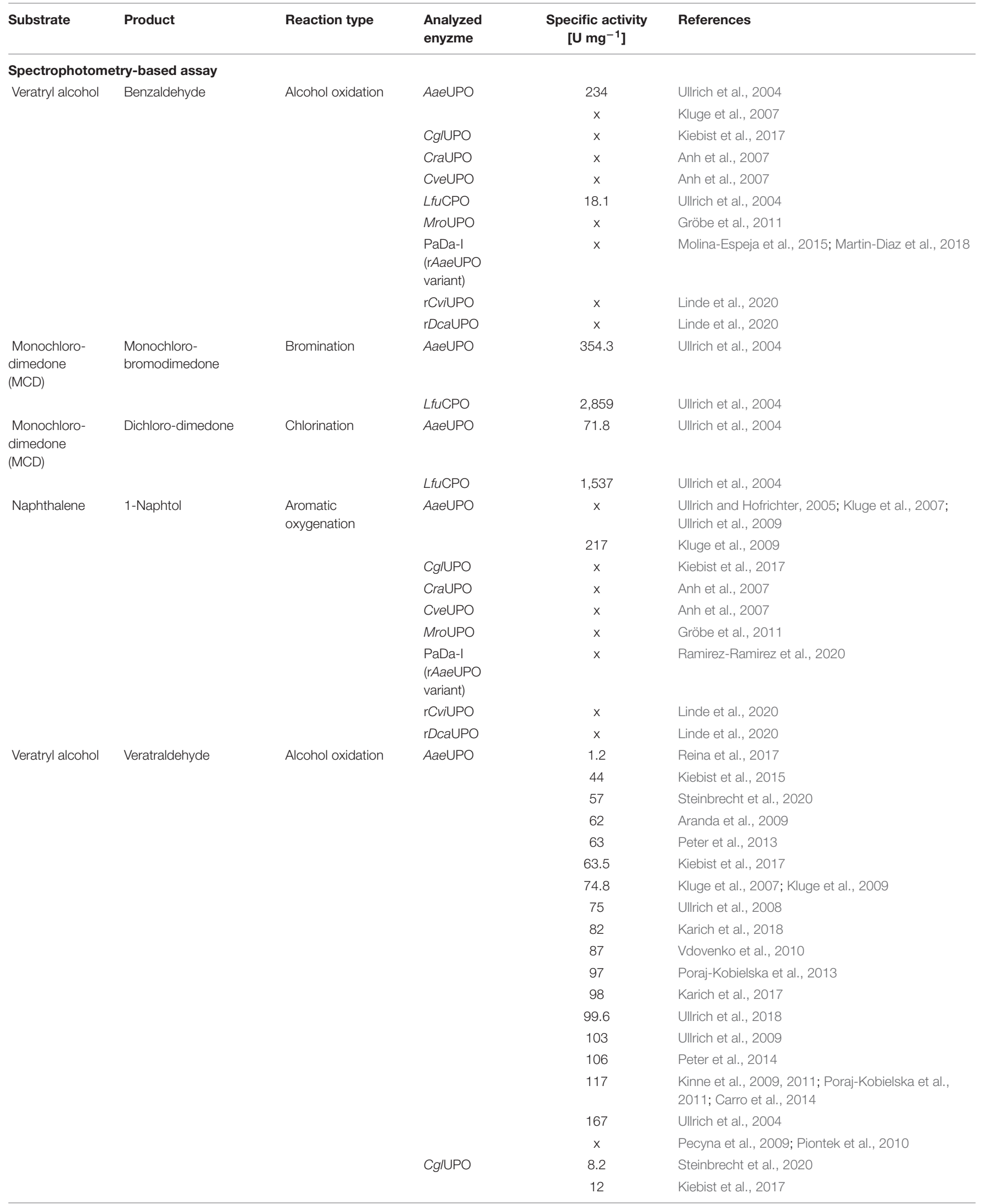


TABLE 3 | Continued

\begin{tabular}{|c|c|c|c|c|c|}
\hline Substrate & Product & Reaction type & $\begin{array}{l}\text { Analyzed } \\
\text { enyzme }\end{array}$ & $\begin{array}{c}\text { Specific activity } \\
{\left[\mathrm{U} \mathrm{mg}^{-1}\right]}\end{array}$ & References \\
\hline \multicolumn{6}{|c|}{ Spectrophotometry-based assay } \\
\hline \multirow[t]{25}{*}{ Veratryl alcohol } & Veratraldehyde & Alcohol oxidation & CraUPO & 23 & Steinbrecht et al., 2020 \\
\hline & & & & 25.8 & Poraj-Kobielska et al., 2011 \\
\hline & & & & 29 & Poraj-Kobielska et al., 2013 \\
\hline & & & & 35 & Aranda et al., 2009 \\
\hline & & & & 38.5 & Anh et al., 2007 \\
\hline & & & CveUPO & $x$ & Anh et al., 2007 \\
\hline & & & MrouPO & 25 & Poraj-Kobielska et al., 2013; Peter et al., 2014 \\
\hline & & & & 26 & Ullrich et al., 2018 \\
\hline & & & & 28 & Karich et al., 2017 \\
\hline & & & & 48.1 & Kiebist et al., 2017 \\
\hline & & & & 61 & Kiebist et al., 2015 \\
\hline & & & & 76 & Gröbe et al., 2011 \\
\hline & & & & 77 & Yarman et al., 2012 \\
\hline & & & & 94 & Steinbrecht et al., 2020 \\
\hline & & & MweUPO & 37.5 & Ullrich et al., 2018 \\
\hline & & & PabUPO & 117 & Kimani, 2019 \\
\hline & & & $\begin{array}{l}\text { PaDa-I } \\
\text { (rAaeUPO } \\
\text { variant) }\end{array}$ & 112 & Horst et al., 2016 \\
\hline & & & & $x$ & $\begin{array}{l}\text { Molina-Espeja et al., 2015; Carro et al., 2018; } \\
\text { Martin-Diaz et al., 2018; Ramirez-Escudero et al., } \\
2018\end{array}$ \\
\hline & & & rCciuPO & 24 & Peter et al., 2014 \\
\hline & & & & 38.18 & Linde et al., 2020 \\
\hline & & & & $\sim 100$ & Babot et al., 2013, 2015; Aranda et al., 2018 \\
\hline & & & & $x$ & González-Benjumea et al., 2020 \\
\hline & & & rDcaUPO & 7.68 & Linde et al., 2020 \\
\hline & & & rHinUPO & 5.4 & Peter et al., 2014 \\
\hline & & & & 15 & Poraj-Kobielska et al., 2013 \\
\hline
\end{tabular}

$x$, substrate accepted.

PaDa-I led to the modification of the peroxidative:peroxygenative ratio activity indicating the coexistence of various oxidation sites in UPOs. The ability to resolve and modify the peroxygenase and peroxidase activities in a targeted manner will certainly allow to diminish the unwanted peroxidase activity opening a new goal for future protein engineering. Of course, one must always keep in mind that the rules of directed evolution apply, i.e., "you get what you screen for" (Schmidt-Dannert and Arnold, 1999). In case of the optimized variant PaDa-I, it turned out that it also oxides thermodynamically inert compounds such as aromatics providing access to naphthalene epoxides that can be subjected to nucleophilic ring opening reactions yielding chiral trans-disubstituted cyclohexane derivatives (Zhang et al., 2021).

However, background absorption of medium compounds like soybean meal, the key inducing ingredient for UPO production in various fungal species, often leads to detection interferences and thus to limitations in the applicability of spectrophotometric assays (Anh et al., 2007; Poraj-Kobielska et al., 2012). In these cases, supplementation with instrumental analysis is often required. Especially if kinetic parameters are to be determined in addition to screenings (Kluge et al., 2007). Furthermore, substrate and product scope of challenging oxyfunctionalization reactions catalyzed by UPOs can be assessed by using GC or HPLC. The AaeUPO turned out to efficiently catalyze the asymmetric benzylic hydroxylation of alkylbenzyl derivatives and $\mathrm{C} 1-\mathrm{C} 2$ epoxidation of styrene derivatives (Kluge et al., 2012). Additionally, the ability to catalyze oxidations at the nitrogen of pyridines is a remarkable property of AaeUPO since heterocyclic $\mathrm{N}$-oxides are desirable biologically active compounds but efficient and convenient synthetic routes are lacking (Ullrich et al., 2008; Mfuh and Larionov, 2015). Smaller libraries consisting of four UPOs were screened for a twostep conversion of cyclohexane into cyclohexanone (Peter et al., 2014). Cyclohexanone is relevant for polymer synthesis or organic solvent. Especially, the second step from cyclohexanol to cyclohexanone turned out to differ substantially dependent on the used UPO. For larger libraries, instrumental analysis must be adapted to screen sufficient numbers of variants generally in the range of up to several thousands. HPLC- and GC-dependent systems generally require long analysis time. The development of efficient screening methods to identify new UPOs of currently uncharacterized strains and to screen mutant libraries is thus 
TABLE 4 | Overview of colorimetry-based UPO assays.

\begin{tabular}{|c|c|c|c|c|c|}
\hline Substrate & Product & Reaction type & $\begin{array}{l}\text { Analyzed } \\
\text { enyzme }\end{array}$ & $\begin{array}{l}\text { Specific activity } \\
{\left[\mathrm{U} \mathrm{mg}^{-1}\right]}\end{array}$ & References \\
\hline \multicolumn{6}{|c|}{ Colorimetry-based assay } \\
\hline \multirow{17}{*}{$\begin{array}{l}2,2^{\prime} \text {-Azinobis-(3- } \\
\text { ethylbenzothiazoline- } \\
\text { 6-sulfonate) } \\
\text { (ABTS) }\end{array}$} & $\begin{array}{l}\text { Green ABTS cation } \\
\text { radical }\end{array}$ & $\begin{array}{l}\text { One-electron } \\
\text { oxidation }\end{array}$ & AaeUPO & 295.7 & Ullrich et al., 2004 \\
\hline & & & & $x$ & $\begin{array}{l}\text { Kluge et al., 2007; Gómez de Santos et al., } \\
2018\end{array}$ \\
\hline & & & CgルPO & $x$ & Kiebist et al., 2017 \\
\hline & & & CraUPO & $x$ & Anh et al., 2007 \\
\hline & & & CveUPO & $x$ & Anh et al., 2007 \\
\hline & & & $\begin{array}{l}\text { JaWa } \\
\text { (rAaeUPO } \\
\text { variant) }\end{array}$ & $x$ & $\begin{array}{l}\text { Molina-Espeja et al., 2016a; Gómez de Santos } \\
\text { et al., } 2018\end{array}$ \\
\hline & & & LfuCPO & 1.2 & Ullich et al., 2004 \\
\hline & & & MroUPO & $x$ & Gröbe et al., 2011 \\
\hline & & & PabUPO & $x$ & Kimani, 2019 \\
\hline & & & $\begin{array}{l}\text { PaDa-I } \\
\text { (rAaeUPO } \\
\text { variant) }\end{array}$ & 607 & Bormann et al., 2020a \\
\hline & & & & 828 & Molina-Espeja et al., 2014 \\
\hline & & & & $x$ & $\begin{array}{l}\text { Molina-Espeja et al., 2015, 2019, 2016a; } \\
\text { Gómez de Santos et al., 2018; Martin-Diaz } \\
\text { et al., 2018; Ramirez-Escudero et al., 2018; } \\
\text { Ramirez-Ramirez et al., } 2020\end{array}$ \\
\hline & & & PaDa-I-Cys & 740 & Molina-Espeja et al., 2019 \\
\hline & & & rAaeUPO & $x$ & Mate et al., 2017; Burek et al., 2019 \\
\hline & & & rCviUPO & 38.2 & González-Benjumea et al., 2020 \\
\hline & & & & $x$ & Linde et al., 2020 \\
\hline & & & rDcaUPO & $x$ & Linde et al., 2020 \\
\hline \multirow{17}{*}{$\begin{array}{l}\text { 2,6- } \\
\text { dimethoxyphenol } \\
\text { (DMP) }\end{array}$} & Colored coerulignone & $\begin{array}{l}\text { One-electron } \\
\text { oxidation }\end{array}$ & AaeUPO & 99.6 & Ullrich et al., 2004 \\
\hline & & & & $x$ & Kluge et al., 2007; Püllmann et al., 2021 \\
\hline & & & CgルPO & $x$ & Kiebist et al., 2017 \\
\hline & & & $\begin{array}{l}\text { JaWa } \\
\text { (rAaeUPO } \\
\text { variant) }\end{array}$ & $x$ & Molina-Espeja et al., 2016a \\
\hline & & & LfuCPO & 1.9 & Ullrich et al., 2004 \\
\hline & & & MroUPO & $x$ & Gröbe et al., 2011 \\
\hline & & & PabUPO & $x$ & Kimani, 2019 \\
\hline & & & $\begin{array}{l}\text { PaDa-I } \\
\text { (rAaeUPO } \\
\text { variant) }\end{array}$ & $x$ & $\begin{array}{l}\text { Molina-Espeja et al., 2014, 2015, 2016a; } \\
\text { Martin-Diaz et al., 2018; Ramirez-Escudero } \\
\text { et al., } 2018\end{array}$ \\
\hline & & & rCg/UPO & $x$ & Püllmann et al., 2021 \\
\hline & & & rDcaUPO & $x$ & Püllmann and Weissenborn, 2021 \\
\hline & & & rGmaUPO & $x$ & Püllmann et al., 2021 \\
\hline & & & rMfeUPO & $x$ & Püllmann and Weissenborn, 2021 \\
\hline & & & rMhiUPO & $x$ & Püllmann and Weissenborn, 2021 \\
\hline & & & rMroUPO & $x$ & Püllmann et al., 2021 \\
\hline & & & rMthUPO & $x$ & Püllmann et al., 2021 \\
\hline & & & rMweUPO & $x$ & Püllmann et al., 2021 \\
\hline & & & rTteUPO & $x$ & Püllmann et al., 2021 \\
\hline
\end{tabular}


TABLE 4 | Continued

\begin{tabular}{|c|c|c|c|c|c|}
\hline Substrate & Product & Reaction type & $\begin{array}{l}\text { Analyzed } \\
\text { enyzme }\end{array}$ & $\begin{array}{l}\text { Specific activity } \\
{\left[\mathrm{U} \mathrm{mg}^{-1}\right]}\end{array}$ & References \\
\hline \multicolumn{6}{|c|}{ Colorimetry-based assay } \\
\hline \multirow{4}{*}{$\begin{array}{l}\text { Naphthalene + Fast } \\
\text { Red reagent [Fast } \\
\text { red TR salt hemi } \\
\text { (zinc chloride)] }\end{array}$} & Red azo dye & $\begin{array}{l}\text { Aromatic } \\
\text { oxygenation }\end{array}$ & $\begin{array}{l}\text { JaWa } \\
\text { (rAaeUPO } \\
\text { variant) }\end{array}$ & $x$ & Molina-Espeja et al., 2016a \\
\hline & & & MroUPO & $x$ & Gröbe et al., 2011 \\
\hline & & & PabUPO & $x$ & Kimani, 2019 \\
\hline & & & $\begin{array}{l}\text { PaDa-I } \\
\text { (rAaeUPO } \\
\text { variant) }\end{array}$ & $x$ & $\begin{array}{l}\text { Molina-Espeja et al., 2016a; Martin-Diaz et al., } \\
2018\end{array}$ \\
\hline \multirow[t]{15}{*}{$\begin{array}{l}\text { 5-nitro-1,3- } \\
\text { benzodioxole } \\
\text { (NBD) }\end{array}$} & $\begin{array}{l}\text { Yellow }(\mathrm{pH} 7) / \text { red } \\
(\mathrm{pH}>12) \\
\text { 4-nitrocatechol }\end{array}$ & Demethylenation & AaeUPO & $x$ & Poraj-Kobielska et al., 2012 \\
\hline & & & Cg/UPO & $x$ & Kiebist et al., 2017 \\
\hline & & & CraUPO & $x$ & Poraj-Kobielska et al., 2012 \\
\hline & & & CveuPO & $x$ & Poraj-Kobielska et al., 2012 \\
\hline & & & $\begin{array}{l}\text { JaWa } \\
\text { (rAaeUPO } \\
\text { variant) }\end{array}$ & $x$ & Molina-Espeja et al., 2016a \\
\hline & & & MroUPO & $x$ & $\begin{array}{l}\text { Poraj-Kobielska et al., 2012; Püllmann et al., } \\
2021\end{array}$ \\
\hline & & & PabUPO & 5.2 & Kimani, 2019 \\
\hline & & & $\begin{array}{l}\text { PaDa-I } \\
\text { (rAaeUPO } \\
\text { variant) }\end{array}$ & $x$ & $\begin{array}{l}\text { Molina-Espeja et al., 2014, 2015, 2019, 2016a; } \\
\text { Gómez de Santos et al., 2018; Martin-Diaz } \\
\text { et al., 2018; Ramirez-Escudero et al., 2018; } \\
\text { Ramirez-Ramirez et al., } 2020\end{array}$ \\
\hline & & & PaDa-I-Cys & 240 & Molina-Espeja et al., 2019 \\
\hline & & & rAaeUPO & $x$ & Mate et al., 2017; Püllmann et al., 2021 \\
\hline & & & rCciuPO & $x$ & $\begin{array}{l}\text { Poraj-Kobielska et al., 2012; Püllmann et al., } \\
2021\end{array}$ \\
\hline & & & rCg/UPO & $x$ & Püllmann et al., 2021 \\
\hline & & & rGmaUPO & $x$ & Püllmann et al., 2021 \\
\hline & & & rMthUPO & $x$ & $\begin{array}{l}\text { Püllmann et al., 2021; Püllmann and } \\
\text { Weissenborn, } 2021\end{array}$ \\
\hline & & & rTteUPO & $x$ & $\begin{array}{l}\text { Püllmann et al., 2021; Püllmann and } \\
\text { Weissenborn, } 2021\end{array}$ \\
\hline
\end{tabular}

$x$, substrate accepted.

needed. An outstanding approach includes a directed-evolution step coupled to a multiple injections in a single experimental run (MISER)-GC-MS-system (Knorrscheidt et al., 2019, 2020). This system enabled a throughput of a 96-well microtiter plate within $50 \mathrm{~min}$ for the analysis of a shuffled library of UPOs heterologously secreted from S. cerevisiae. Overall, 672 variants were screened within $7 \mathrm{~h}$ on their activity toward 1,2,3,4tetrahydronaphthalene oxidation resulting the identification of more than 30 novel mutant hits.

While there are a few examples available screening UPO mutant libraries based on heterologous expression systems, there are no high-throughput approaches yet performed to analyze putative UPO-producing wild-type fungi and thus the potential that nature provides us. The natural function of UPOs remains unclear, with different activities proposed, including metabolite synthesis, lignin degradation, and detoxification processes (Hofrichter et al., 2015). Up to now, nine ascomycetous and eight basidiomycetous UPOs were characterized corresponding to $0.4 \%$ compared to the uncharacterized putative UPO sequences. In general, compared to high-throughput processes for bacteria and yeast, there has been less progress in developing and validating screening assays for filamentous fungi (RothschildMancinelli et al., 2020). High-throughput screening of not commonly used fungi can have several bottlenecks. These are mainly, amongst others, the non-homogeneous growth in liquid media due to mycelium or pellet formation, low protein production, dependence of protein production on growth condition and medium compounds (Hofrichter et al., 2020; Rothschild-Mancinelli et al., 2020). Nevertheless, screening approaches for fungi were performed for microbial strain libraries containing bacteria and fungi, which were screened on monooxygenase activity using pharmaceutical compounds analyzed with LC-MS (Fredenhagen et al., 2019; Schmitz et al., 2019b). This screening approach is thus available to be 


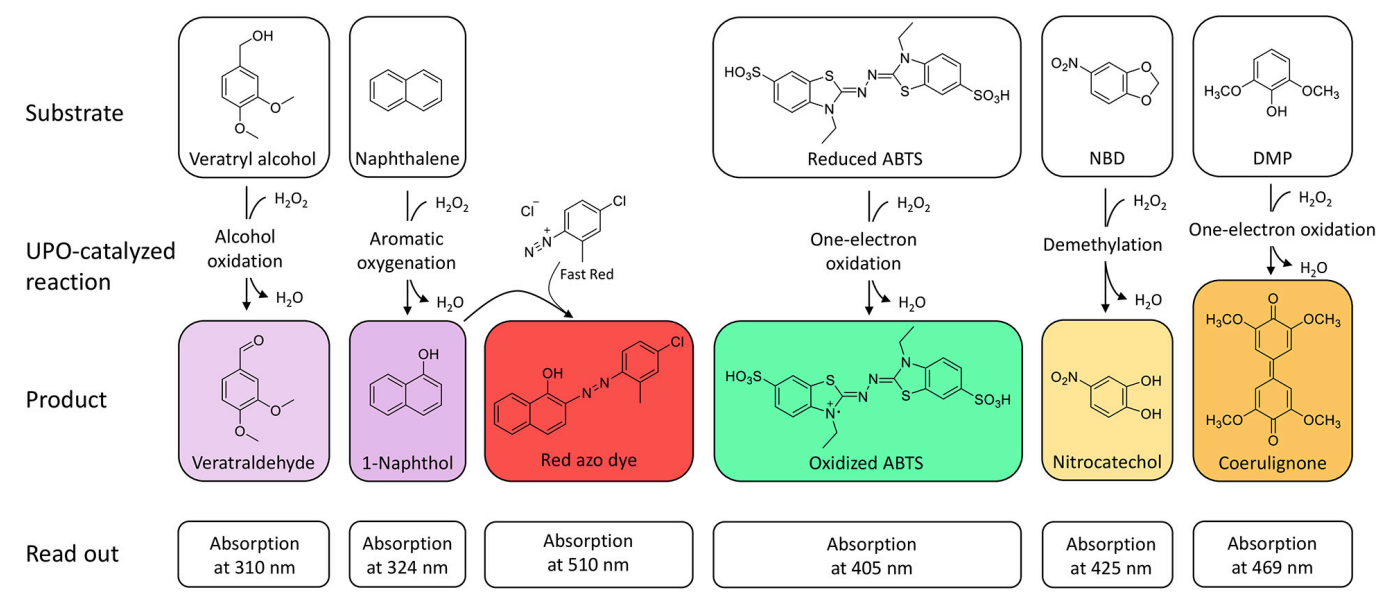

FIGURE 2 | Spectrophotometric assays for the detection and quantification of UPO activity. The products veratraldehyde and 1-naphthol absorb in the non-visible range. Oxidized ABTS, nitrocatechol and coerulignone are visible and can also be used in colorimetric assays.

transferred to peroxygenase activity screenings in the future, which would be a first step toward medium- to high-throughput screening of natural UPO producing strains. Miniaturization in microwell plate format and automated expression screening has also been successfully performed with filamentous fungi (Alberto et al., 2009). The use of microfluidics and microdroplets can further increase throughput to test millions of biocatalyst candidates. For example, enzyme containing solution or the enzyme producing organism and substrate can be encapsulated in microdroplets and then analyzed with fluorescenceactivated sorting methods (Beneyton et al., 2016). Droplet microfluidics combined with electrospray ionization (ESI)MS can also provide label-free, high-throughput screening with the opportunity to test simultaneously substrate libraries (Diefenbach et al., 2018).

\section{CONCLUSION}

Fungal UPOs catalyze both, one-electron oxidations (typical peroxidase reactions) and two-electron oxidations with peroxidederived oxygen transfer (peroxygenation reactions), the latter being more interesting with respect to synthesis of pharmaceuticals. The co-existence of both activities in one biocatalyst, however, leads to lower yields, for instance, due to one-electron oxidation of the desired hydroxylated product. This issue has been successfully addressed by directed evolution in combination with the addition of radical scavengers (MolinaEspeja et al., 2016a; Gómez de Santos et al., 2018). In context of synthesis of phenolic products like $5^{\prime}$-hydroxypropranolol or $4^{\prime}$-hydroxydiclofenac, ascorbic acid has often been used as a radical scavenger since it reduces the formation of unwanted side-products through its oxidation into ascorbyl radical and simultaneous re-reduction of the intermediate phenoxyl radical (Ullrich and Hofrichter, 2007; Poraj-Kobielska et al., 2013; Gómez de Santos et al., 2018). Up to now, only very few approaches have been able to express UPOs at titers in the "gper-liter" range (Hofrichter et al., 2020). Low stability of UPOs against $\mathrm{H}_{2} \mathrm{O}_{2}$ is typical for all heme-containing enzymes and can be overcome by constant feeding of $\mathrm{H}_{2} \mathrm{O}_{2}$ (Zhang et al., 2017), in situ $\mathrm{H}_{2} \mathrm{O}_{2}$ generation (Churakova et al., 2011), or using milder organic peroxides as an alternative (Fernández-Fueyo et al., 2016). Moreover, immobilization strategies have successfully been employed to improve UPO-catalyzed biotransformation through reusability, long-term storage of the enzyme in e.g., cyclohexane, and thus lower cost contribution (Poraj-Kobielska et al., 2015; Fernández-Fueyo et al., 2016; Bormann et al., 2020a). However, UPOs are still in their infancy on the road to large-scale industrial application, and both research and optimization are needed to turn promiscuous enzymes into feasible biocatalysts (Rosenthal and Lütz, 2018; Hobisch et al., 2020; Aranda et al., 2021). In addition to wet lab analysis, computational simulation-based techniques can be used to model and even predict UPO-catalyzed bioprocesses, e.g., in terms of hydrogen peroxide feeding rate (Bormann et al., 2020b; Municoy et al., 2020). Consequently, a combined computationalexperimental approach can make enzyme screening more time-saving and efficient.

Regarding the optimization of UPO-catalyzed biotransformations, sustainability and the environmental impact of biochemical reactions are also increasingly coming into focus and gaining importance for process development. Tieves et al. (2019) analyzed the recombinant expression of evolved rAaeUPO PaDa-I by $P$. pastoris coupled with the in situ $\mathrm{H}_{2} \mathrm{O}_{2}$ generation by the format oxidase AoFOx from A. oryzae, expressed by E. coli, and evaluated the bienzymatic cascade for hydroxylation of ethyl benzene to (R)-1-phenyl ethanol with respect to the commonly used Sheldon's E-factor (E for environmental; Sheldon, 2007) as well as the $\mathrm{E}^{+}$-factor (classical E-factor plus $\mathrm{CO}_{2}$-emissions caused by electricity generation). As a result, their calculation led to an E-factor of $4,300 \mathrm{~kg}_{\text {waste }} \mathrm{kg}_{\text {product }}{ }^{-1}$ for crude enzyme and $18,500 \mathrm{~kg}_{\text {waste }} \mathrm{kg}_{\text {product }}{ }^{-1}$ for the purified UPO. Including electricity-related $\mathrm{CO}_{2}$ emissions, the $\mathrm{E}^{+}$-factors even reached 566,800 $\mathrm{kg}_{\text {waste }} \mathrm{kg}_{\text {product }}{ }^{-1}$ most likely due to resource-consuming purification steps. As the turnover number (TN) and the protein yield are powerful key factors for the 
environmental impact of a biocatalyst, increasing the TN of $\mathrm{rAaeUPO}$ and optimization of the enzyme preparation process can significantly imply lower E-factor contribution (Tieves et al., 2019). Moreover, Bello et al. (2020) recently presented a life cycle assessment (LCA) study of the production and purification of CglUPO in its native producer C. globosum for the enzymatic production of 2,5-furandicarboxylic acid (FDCA) as precursor of bioplastics. Their extensive analysis by impact categories, ranging from land use, human toxicity and ecotoxicity to ozone depletion and global warming, clearly indicated that the main environmental impacts can be attributed to electricity, glucose consumption and culture medium chemicals on laboratory (6 L) and large scale (100,000 L). Among others, polluting or harmful compounds, such as ammonia, nitrates, or methanol, and energy-intensive processes rooted from coal were included

\section{REFERENCES}

Alberto, F., Navarro, D., De Vries, R. P., Asther, M., and Record, E. (2009). 'Technical advance in fungal biotechnology: development of a miniaturized culture method and an automated high-throughput screening'. Lett. Appl. Microbiol. 49, 278-282. doi: 10.1111/j.1472-765X.2009.02655.x

Anh, D. H., Ullrich, R., Benndorf, D., Svatoś, A., Muck, A., and Hofrichter, M. (2007). 'The coprophilous mushroom Coprinus radians secretes a haloperoxidase that catalyzes aromatic peroxygenation'. Appl. Environ. Microbiol. 73, 5477-5485. doi: 10.1128/AEM.00026-07

Aranda, C., Carro, J., González-Benjumea, A., Babot, E. D., Olmedo, A., Linde, D., et al. (2021). Advances in enzymatic oxyfunctionalization of aliphatic compounds. Biotechnol. Adv. doi: 10.1016/j.biotechadv.2021.107703 [Epub ahead of print].

Aranda, C., Ullrich, R., Kiebist, J., Scheibner, K., Del Río, J. C., Hofrichter, M., et al. (2018). 'Selective synthesis of the resveratrol analogue 4,4'-dihydroxytrans-stilbene and stilbenoids modification by fungal peroxygenases'. Catal. Sci. Technol. 8, 2394-2401. doi: 10.1039/c8cy00272j

Aranda, E., Kinne, M., Kluge, M., Ullrich, R., and Hofrichter, M. (2009). 'Conversion of dibenzothiophene by the mushrooms Agrocybe aegerita and Coprinellus radians and their extracellular peroxygenases'. Appl. Microbiol. Biotechnol. 82, 1057-1066. doi: 10.1007/s00253-008-1778-6

Babot, E. D., del Río, J. C., Kalum, L., Martínez, A. T., and Gutiérrez, A. (2013). 'Oxyfunctionalization of aliphatic compounds by a recombinant peroxygenase from Coprinopsis cinerea'. Biotechnol. Bioeng. 110, 2323-2332. doi: 10.1002/bit. 24904

Babot, E. D., Río, C., Kalum, L., Martínez, A. T., and Gutierrez, A. (2015). 'Regioselective hydroxylation in the production of 25-Hydroxyvitamin D by Coprinopsis cinerea peroxygenase'. ChemCatChem 7, 283-290. doi: 10.1002/ cctc. 201402795

Bello, S., Pérez, N., Kiebist, J., Scheibner, K., Sánchez Ruiz, M. I., Serrano, A., et al. (2020). Early-stage sustainability assessment of enzyme production in the framework of lignocellulosic biorefinery. J. Clean. Prod. 285:125461. doi: 10.1016/j.jclepro.2020.125461

Beneyton, T., Wijaya, I. P. M., Postros, P., Najah, M., Leblond, P., Couvent, A., et al. (2016). High-throughput screening of filamentous fungi using nanoliter-range droplet-based microfluidics. Sci. Rep. 6:27223. doi: 10.1038/srep27223

Bormann, S., Burek, B. O., Ulber, R., and Holtmann, D. (2020a). Immobilization of unspecific peroxygenase expressed in Pichia pastoris by metal affinity binding. Mol. Catal. 492:110999. doi: 10.1016/j.mcat.2020.110999

Bormann, S., Gomez Baraibar, A., Ni, Y., Holtmann, D., and Hollmann, F. (2015). 'Specific oxyfunctionalisations catalysed by peroxygenases: opportunities, challenges and solutions'. Catal. Sci. Technol. 5, 2038-2052. doi: 10.1039/ c4cy01477d

Bormann, S., Hertweck, D., Schneider, S., Bloh, J. Z., Ulber, R., Spiess, A. C., et al. (2020b). 'Modeling and simulation-based design of electroenzymatic batch processes catalyzed by unspecific peroxygenase from A. aegerita'. Biotechnol. Bioeng. 118, 7-16. doi: 10.1002/bit.27545 in the calculation, revealing critical bottlenecks of the UPO production process (Bello et al., 2020). In summary, studies such as these highlight the importance of identification and characterization of novel UPO enzymes, protein engineering combined with medium- to high-throughput screenings as well as process optimization approaches in both economic and environmental terms.

\section{AUTHOR CONTRIBUTIONS}

AK, KR, and SL conceptualized and designed the manuscript. AK organized the literature research. AK and KR wrote the manuscript. All authors contributed to manuscript revision, read, and approved the submitted version.

Breslmayr, E., Hanžek, M., Hanrahan, A., Leitner, C., Kittl, R., Šantek, B., et al. (2018). 'A fast and sensitive activity assay for lytic polysaccharide monooxygenase'. Biotechnol. Biofuels 11, 1-13. doi: 10.1186/s13068-018-10636

Buchhaupt, M., Ehrich, K., Hüttmann, S., Guder, J., and Schrader, J. (2011). 'Overexpression of chloroperoxidase in Caldariomyces fumago'. Biotechnol. Lett. 33, 2225-2231. doi: 10.1007/s10529-011-0683-8

Buchhaupt, M., Hüttmann, S., and Schrader, J. (2012). 'White mutants of chloroperoxidase-secreting caldariomyces fumago as superior production strains, revealing an interaction between pigmentation and enzyme secretion'. Appl. Environ. Microbiol. 78, 5923-5925. doi: 10.1128/AEM.00570-12

Burek, B. O., de Boer, S. R., Tieves, F., Zhang, W., van Schie, M., Bormann, S., et al. (2019). 'Photoenzymatic hydroxylation of ethylbenzene catalyzed by unspecific peroxygenase: origin of enzyme inactivation and the impact of light intensity and temperature'. ChemCatChem 11, 3093-3100. doi: 10.1002/cctc.2019 00610

Carro, J., Fernández-Fueyo, E., Fernández-Alonso, C., Cañada, J., Ullrich, R., Hofrichter, M., et al. (2018). 'Self-sustained enzymatic cascade for the production of 2,5-furandicarboxylic acid from 5-methoxymethylfurfural'. Biotechnol. Biofuels 11:86. doi: 10.1186/s13068-018-1091-2

Carro, J., Ferreira, P., Rodríguez, L., Prieto, A., Serrano, A., Balcells, B., et al. (2014). '5-Hydroxymethylfurfural conversion by fungal aryl-alcohol oxidase and unspecific peroxygenase'. FEBS J. 282, 3218-3229. doi: 10.1111/febs.13177

Carro, J., González-Benjumea, A., Fernández-Fueyo, E., Aranda, C., Guallar, V., Gutiérrez, A., et al. (2019). 'Modulating fatty acid epoxidation vs hydroxylation in a fungal peroxygenase'. ACS Catal. 9, 6234-6242. doi: 10.1021/acscatal. 9 b01454

Churakova, E., Kluge, M., Ullrich, R., Arends, I., Hofrichter, M., and Hollmann, F. (2011). 'Specific photobiocatalytic oxyfunctionalization reactions'. Angew. Chem. Int. Ed. Engl. 50, 10716-10719. doi: 10.1002/anie.201105308

Conesa, A., Van De Velde, F., Van Rantwijk, F., Sheldon, R. A., Van Den Hondel, C. A., and Punt, P. J. (2001). 'Expression of the Caldariomyces fumago chloroperoxidase in Aspergillus niger and characterization of the recombinant enzyme'. J. Biol. Chem. 276, 17635-17640. doi: 10.1074/jbc.M0105 71200

Demain, A. L., and Vaishnav, P. (2009). 'Production of recombinant proteins by microbes and higher organisms'. Biotechnol. Adv. 27, 297-306. doi: 10.1016/j. biotechadv.2009.01.008

Diefenbach, X. W., Farasat, I., Guetschow, E. D., Welch, C. J., Kennedy, R. T., Sun, S., et al. (2018). 'Enabling biocatalysis by high-throughput protein engineering using droplet microfluidics coupled to mass spectrometry'. ACS Omega 3, 1498-1508. doi: 10.1021/acsomega.7b01973

Durand, H., Clanet, M., and Tiraby, G. (1988). 'Genetic improvement of Trichoderma reesei for large scale cellulase production'. Enzyme Microb. Technol. 10, 341-346. doi: 10.1016/0141-0229(88)90012-9

Faiza, M., Huang, S., Lan, D., and Wang, Y. (2019a). 'New insights on unspecific peroxygenases: superfamily reclassification and evolution'. BMC Evol. Biol. 19, 1-19. doi: 10.1186/s12862-019-1394-3 
Faiza, M., Lan, D., Huang, S., and Wang, Y. (2019b). 'UPObase: an online database of unspecific peroxygenases'. Database 2019:baz122. doi: 10.1093/database/ baz122

Fernández-Fueyo, E., Aranda Oliden, C., Gutiérrez Suárez, A., and Martånez Ferrer, A. T. (2019). 'Method of Heterologous Expression of Active Fungal Unspecific Peroxygenase in Bacterial Host Cells for Fatty-Acid Epoxidation and Other Oxygenation Reactions'. Patent (international): WO 2020/011847 A1

Fernández-Fueyo, E., Ni, Y., Gomez Baraibar, A., Alcalde, M., van Langen, L. M., and Hollmann, F. (2016). 'Towards preparative peroxygenase-catalyzed oxyfunctionalization reactions in organic media'. J. Mol. Catal. B: Enzym. 134, 347-352. doi: 10.1016/j.molcatb.2016.09.013

Fredenhagen, A., Schroer, K., Schröder, H., Hoepfner, D., Ligibel, M., Porchet Zemp, L., et al. (2019). 'Cladosporin derivatives obtained by biotransformation provide guidance for the focused derivatization of this antimalarial lead compound'. ChemBioChem 20, 650-654. doi: 10.1002/cbic.201800588

Geueke, B., and Kohler, H.-P. E. (2010). "Enzyme assays, substrate specificities, kinetic parameters: measurement of enzyme activities," in Handbook of Hydrocarbon and Lipid Microbiology, ed. K. N. Timmis (Berlin: Springer), 4195-4202. doi: 10.1007/978-3-540-77587-4_327

Gómez de Santos, P., Cañellas, M., Tieves, F., Younes, S. H. H., Molina-Espeja, P., Hofrichter, M., et al. (2018). 'Selective synthesis of the human drug metabolite 5'-hydroxypropranolol by an evolved self-sufficient peroxygenase'. ACS Catal. 8, 4789-4799. doi: 10.1021/acscatal.8b01004

Gómez de Santos, P., Cervantes, F. V., Tieves, F., Plou, F. J., Hollmann, F., and Alcalde, M. (2019). 'Benchmarking of laboratory evolved unspecific peroxygenases for the synthesis of human drug metabolites'. Tetrahedron 75, 1827-1831. doi: 10.1016/j.tet.2019.02.013

González-Benjumea, A., Carro, J., Renau-Mínguez, C., Linde, D., FernándezFueyo, E., Gutiérrez, A., et al. (2020). 'Fatty acid epoxidation by Collariella virescens peroxygenase and heme-channel variants'. Catal. Sci. Technol. 10, 717-725. doi: 10.1039/c9cy02332a

Gonzalez-Perez, D., Molina-Espeja, P., Garcia-Ruiz, E., and Alcalde, M. (2014). 'Mutagenic Organized Recombination Process by Homologous In vivo Grouping (MORPHING) for directed enzyme evolution'. PLoS One 9:e90919. doi: 10.1371/journal.pone.0090919

Gröbe, G., Ullrich, R., Pecyna, M. J., Kapturska, D., Friedrich, S., Hofrichter, M., et al. (2011). 'High-yield production of aromatic peroxygenase by the agaric fungus Marasmius rotula'. AMB Express 1:31. doi: 10.1186/2191-0855-1-31

Herzog, R., Solovyeva, I., Bölker, M., Lugones, L. G., and Hennicke, F. (2019). 'Exploring molecular tools for transformation and gene expression in the cultivated edible mushroom Agrocybe aegerita'. Mol. Genet. Genom. 294, 663677. doi: 10.1007/s00438-018-01528-6

Hobisch, M., Holtmann, D., Gómez de Santos, P., Alcalde, M., Hollmann, F., and Kara, S. (2020). Recent developments in the use of peroxygenases - exploring their high potential in selective oxyfunctionalisations. Biotechnol. Adv. doi: 10.1016/j.biotechadv.2020.107615 [Epub ahead of print].

Hofrichter, M., Kellner, H., Herzog, R., Karich, A., Liers, C., Scheibner, K., et al. (2020). "Fungal peroxygenases: a phylogenetically old superfamily of heme enzymes with promiscuity for oxygen transfer reactions," in Grand Challenges in Fungal Biotechnology, ed. H. Nevalainen (Berlin: Springer), 369-403. doi: 10.1007/978-3-030-29541-7_14

Hofrichter, M., Kellner, H., Pecyna, M. J., and Ullrich, R. (2015). "fungal unspecific peroxygenases: heme-thiolate proteins that combine peroxidase and cytochrome $\mathrm{P} 450$ properties," in Monooxygenase, Peroxidase and Peroxygenase Properties and Mechanisms of Cytochrome P450, eds E. G. Hrycay and S. M. Bandiera (Berlin: Springer), 341-368. doi: 10.1007/978-3-319-16009-2

Hofrichter, M., Scheibner, K., Nüske, J., and Ullrich, R. (2005). 'Method for the Enzymatic Hydroxylation of Non-Activated Hydrocarbons'. Patent (international): WO 2006/034702 A1

Hofrichter, M., Scheibner, K., Ullrich, R., Kinne, M., Peter, S., Lund, H., et al. (2011). 'Enzymatic Hydroxylation of Aliphatic Hydrocarbon'. Patent (international): WO 2011/120938 A2

Holla, W., Atzrodt, J., Sandvoss, M., Heidrich, J., Scheibner, K., Gröbe, G., et al. (2015). 'Process for Peroxygenase-Catalyzed Cleavage of Benzyloxycarbonyl Protective Groups'. Patent (European): EP 3128012 A1

Horst, A. E. W., Bormann, S., Meyer, J., Steinhagen, M., Ludwig, R., Drews, A., et al. (2016). 'Electro-enzymatic hydroxylation of ethylbenzene by the evolved unspecific peroxygenase of Agrocybe aegerita'. J. Mol. Catal. B Enzym. 133, S137-S142. doi: 10.1016/j.molcatb.2016.12.008

Johannes, T. W., Woodyer, R. D., and Zhao, H. (2006). "High-throughput screening methods developed for oxidoreductases," in Enzyme Assays: HighThroughput Screening, Genetic Selection and Fingerprinting, ed. J.-L. Reymond (Hoboken, NJ: Wiley Blackwell), 77-93. doi: 10.1002/3527607846.ch3

Karich, A., Kleeberg, S., Ullrich, R., and Hofrichter, M. (2018). 'Enzymatic preparation of 2,5-Furandicarboxylic Acid (FDCA)-a substitute of terephthalic acid-by the joined action of three fungal enzymes'. Microorganisms 6:5. doi: 10.3390/microorganisms6010005

Karich, A., Scheibner, K., Ullrich, R., and Hofrichter, M. (2016). 'Exploring the catalase activity of unspecific peroxygenases and the mechanism of peroxidedependent heme destruction'. J. Mol. Catal. B Enzym. 134, 238-246. doi: 10. 1016/j.molcatb.2016.10.014

Karich, A., Ullrich, R., Scheibner, K., and Hofrichter, M. (2017). Fungal unspecific peroxygenases oxidize the majority of organic EPA priority pollutants. Front. Microbiol. 8:1463. doi: 10.3389/fmicb.2017.01463

Kazlauskas, R. J. (2006). "Quantitative assay of hydrolases for activity and selectivity using color changes," in Enzyme Assays: High-Throughput Screening, Genetic Selection and Fingerprinting, ed. J.-L. Reymond (Hoboken, NJ: Wiley Blackwell), 15-39. doi: 10.1002/3527607846.ch1

Kellner, H., Luis, P., Pecyna, M. J., Barbi, F., Kapturska, D., Krugg̈er, D., et al. (2014). Widespread occurrence of expressed fungal secretory peroxidases in forest soils. PLoS One 9:e95557. doi: 10.1371/journal.pone.0095557

Kiebist, J., Holla, W., Heidrich, J., Poraj-Kobielska, M., Sandvoss, M., Simonis, R., et al. (2015). 'One-pot synthesis of human metabolites of SAR548304 by fungal peroxygenases'. Bioorg. Med. Chem. 23, 4324-4332. doi: 10.1016/j.bmc.2015.06. 035

Kiebist, J., Schmidtke, K. U., Zimmermann, J., Kellner, H., Jehmlich, N., Ullrich, R., et al. (2017). 'A peroxygenase from chaetomium globosum catalyzes the selective oxygenation of testosterone'. ChemBioChem 18,563-569. doi: 10.1002/ cbic. 201600677

Kimani, V. W. (2019). New Secretory Peroxidases and Peroxygenases from Saprotrophic Fungi of Kenyan Forests. Ph.D. thesis. Dresden: TU Dresden.

Kinne, M., Poraj-Kobielska, M., Ralph, S. A., Ullrich, R., Hofrichter, M., and Hammel, K. E. (2009). 'Oxidative cleavage of diverse ethers by an extracellular fungal peroxygenase’. J. Biol. Chem. 284, 29343-29349. doi: 10.1074/jbc.M109. 040857

Kinne, M., Poraj-Kobielska, M., Ullrich, R., Nousiainen, P., Sipilä, J., Scheibner, K., et al. (2011). 'Oxidative cleavage of non-phenolic $\beta$-O-4 lignin model dimmers by an extracellular aromatic peroxygenase'. Holzforschung 65, 673-679. doi: 10.1515/HF.2011.057

Kluge, M., Ullrich, R., Dolge, C., Scheibner, K., and Hofrichter, M. (2009). 'Hydroxylation of naphthalene by aromatic peroxygenase from Agrocybe aegerita proceeds via oxygen transfer from $\mathrm{H} 2 \mathrm{O} 2$ and intermediary epoxidation'. Appl. Microbiol. Biotechnol. 81, 1071-1076. doi: 10.1007/s00253008-1704-y

Kluge, M., Ullrich, R., Scheibner, K., and Hofrichter, M. (2012). 'Stereoselective benzylic hydroxylation of alkylbenzenes and epoxidation of styrene derivatives catalyzed by the peroxygenase of Agrocybe aegerita'. Green Chem. 14, 440-446. doi: $10.1039 / \mathrm{clgc} 16173 \mathrm{c}$

Kluge, M. G., Ullrich, R., Scheibner, K., and Hofrichter, M. (2007). 'Spectrophotometric assay for detection of aromatic hydroxylation catalyzed by fungal haloperoxidase-peroxygenase'. Appl. Microbiol. Biotechnol. 75, 1473-1478. doi: 10.1007/s00253-007-0942-8

Knorrscheidt, A., Püllmann, P., Schell, E., Freier, E., and Weissenborn, M. J. (2020). 'Identification of novel unspecific peroxygenase chimeras and unusual YfeX axial heme ligand by a versatile high-throughput GC-MS approach'. ChemCatChem 12, 4788-4795. doi: 10.1002/cctc.202000618

Knorrscheidt, A., Püllmann, P., Schell, E., Homann, D., Freier, E., and Weissenborn, M. (2019). Development of 96 multiple injection-GC-MS technique and its application in protein engineering of natural and non-natural enzymatic reactions. ChemRxiv [Preprint]. doi: 10.26434/chemrxiv.10314239

Knorrscheidt, A., Soler, J., Hünecke, N., Püllmann, P., Garcia-Borràs, M., and Weissenborn, M. J. (2021). Simultaneous screening of multiple substrates with an unspecific peroxygenase enabled modified alkane and alkene oxyfunctionalisations. Catal. Sci. Technol. doi: 10.1039/D0CY02457K 
Landvik, S., Østergaard, L.-H., and Kalum, L. (2013). 'Polypeptides Having Peroxygenase Activity'. Patent (international): WO 2014/056920 A2

Li, D., Tang, Y., Lin, J., and Cai, W. (2017). 'Methods for genetic transformation of filamentous fungi'. Microb. Cell Fact. 16:168. doi: 10.1186/s12934-017-0785-7

Lichius, A., Ruiz, D. M., and Zeilinger, S. (2020). "Genetic transformation of filamentous fungi: achievements and challenges," in Grand Challenges in Fungal Biotechnology, ed. H. Nevalainen (Berlin: Springer), 123-164. doi: 10.1007/9783-030-29541-7_5

Linde, D., Olmedo, A., González-Benjumea, A., Estévez, M., Renau-Mínguez, C., Carro, J., et al. (2020). 'Two new unspecific peroxygenases from heterologous expression of fungal genes in Escherichia coli. Appl. Environ. Microbiol. 86, 1-16. doi: 10.1128/AEM.02899-19

Lütz, S., Steckhan, E., and Liese, A. (2004). 'First asymmetric electroenzymatic oxidation catalyzed by a peroxidase'. Electrochem. Commun. 6, 583-587. doi: 10.1016/j.elecom.2004.04.009

Martin-Diaz, J., Paret, C., García-Ruiz, E., Molina-Espeja, P., and Alcalde, M. (2018). 'Shuffling the neutral drift of unspecific peroxygenase in Saccharomyces cerevisiae'. Appl. Environ. Microbiol. 84, 1-12. doi: 10.1128/AEM.00808-18

Mate, D. M., Palomino, M. A., Molina-Espeja, P., Martin-Diaz, J., and Alcalde, M. (2017). 'Modification of the peroxygenative: peroxidative activity ratio in the unspecific peroxygenase from Agrocybe aegerita by structure-guided evolution'. Protein Eng. Des. Sel. 30, 191-198. doi: 10.1093/protein/gzw073

Mfuh, A. M., and Larionov, O. V. (2015). 'Heterocyclic N-Oxides - an emerging class of therapeutic agents'. Curr. Med. Chem. 22, 2819-2857. doi: 10.2174/ 0929867322666150619104007

Molina-Espeja, P., Cañellas, M., Plou, F. J., Hofrichter, M., Lucas, F., Guallar, V., et al. (2016a). 'Synthesis of 1-Naphthol by a natural peroxygenase engineered by directed evolution'. ChemBioChem 17, 341-349. doi: 10.1002/cbic.201500493

Molina-Espeja, P., Garcia-Ruiz, E., Gonzalez-Perez, D., Ullrich, R., Hofrichter, M., and Alcalde, M. (2014). 'Directed evolution of unspecific peroxygenase from Agrocybe aegerita'. Appl. Environ. Microbiol. 80, 3496-3507. doi: 10.1128/AEM. 00490- 14

Molina-Espeja, P., Ma, S., Mate, D. M., Ludwig, R., and Alcalde, M. (2015). 'Tandem-yeast expression system for engineering and producing unspecific peroxygenase'. Enzyme Microb. Technol. 7, 29-33. doi: 10.1016/j.enzmictec. 2015.03.004

Molina-Espeja, P., Plou Gasca, F. J., Alcalde Galeote, M., and Gómez de Santos, P. (2016b). 'Mutants of Unspecific Peroxygenase with High Monooxygenase Activity and Uses Thereof'. Patent (international): WO 2017/081355 Al

Molina-Espeja, P., Santos-Moriano, P., García-Ruiz, E., Ballesteros, A., Plou, F. J., and Alcalde, M. (2019). 'Structure-guided immobilization of an evolved unspecific peroxygenase'. Int. J. Mol. Sci. 20, 1-12. doi: 10.3390/ijms20071627

Municoy, M., González-Benjumea, A., Carro, J., Aranda, C., Linde, D., RenauMínguez, C., et al. (2020). 'Fatty-acid oxygenation by fungal peroxygenases: from computational simulations to preparative regio: from stereoselective epoxidation'. ACS Catal. 10, 13584-13595. doi: 10.1021/acscatal.0c0 3165

Ni, Y., Fernández-Fueyo, E., Baraibar, A. G., Ullrich, R., Hofrichter, M., Yanase, H., et al. (2016). 'Peroxygenase-catalyzed oxyfunctionalization reactions promoted by the complete oxidation of methanol'. Angew. Chem. Int. Ed. Engl. 55, 798-801. doi: 10.1002/anie.201507881

Olmedo, A., Río, J. C., Kiebist, J., Ullrich, R., Hofrichter, M., Scheibner, K., et al. (2017). 'Fatty acid chain shortening by a fungal peroxygenase'. Chemistry 23, 16985-16989. doi: 10.1002/chem.201704773

Pecyna, M. J., Schnorr, K. M., Ullrich, R., Scheibner, K., Kluge, M. G., and Hofrichter, M. (2008). 'Fungal Peroxygenases and Methods of Application'. Patent (international): WO 2008/119780 A2

Pecyna, M. J., Ullrich, R., Bittner, B., Clemens, A., Scheibner, K., Schubert, R., et al. (2009). 'Molecular characterization of aromatic peroxygenase from Agrocybe aegerita'. Appl. Microbiol. Biotechnol. 84, 885-897. doi: 10.1007/s00253-0092000-1

Peter, S., Karich, A., Ullrich, R., Gröbe, G., Scheibner, K., and Hofrichter, M. (2014). 'Enzymatic one-pot conversion of cyclohexane into cyclohexanone: comparison of four fungal peroxygenases'. J. Mol. Catal. B Enzym. 103, 47-51. doi: 10.1016/j.molcatb.2013.09.016

Peter, S., Kinne, M., Ullrich, R., Kayser, G., and Hofrichter, M. (2013). 'Epoxidation of linear, branched and cyclic alkenes catalyzed by unspecific peroxygenase'. Enzyme Microb. Technol. 52, 370-376. doi: 10.1016/j.enzmictec.2013.02.013
Pickard, M. A. (1981). 'A defined growth medium for the production of chloroperoxidase by Caldariomyces fumago'. Can. J. Microbiol. 27, 1298-1305. doi: $10.1139 / \mathrm{m} 81-199$

Piontek, K., Strittmatter, E., and Plattner, D. A. (2017). Crystallization of a Dimeric Heme Peroxygenase. Available online at: https://www.rcsb.org/structure/5FUJ (accessed April 8 April, 2021)

Piontek, K., Strittmatter, E., Ullrich, R., Gröbe, G., Pecyna, M. J., Kluge, M., et al. (2013). 'Structural basis of substrate conversion in a new aromatic peroxygenase: cytochrome P450 functionality with benefits'. J. Biol. Chem. 288, 34767-34776. doi: 10.1074/jbc.M113.514521

Piontek, K., Ullrich, R., Liers, C., Diederichs, K., Plattner, D. A., and Hofrichter, M. (2010). 'Crystallization of a $45 \mathrm{kDa}$ peroxygenase/peroxidase from the mushroom Agrocybe aegerita and structure determination by SAD utilizing only the haem iron'. Acta Crystallogr. Sect. F Struct. Biol. Cryst. Commun. 66, 693-698. doi: 10.1107/S1744309110013515

Poraj-Kobielska, M., Atzrodt, J., Holla, W., Sandvoss, M., Gröbe, G., Scheibner, K., et al. (2013). 'Preparation of labeled human drugmetabolites and drug-drug interaction-probes with fungal peroxygenases'. J. Labelled Comp. Radiopharm. 56, 513-519. doi: 10.1002/jlcr.3103

Poraj-Kobielska, M., Kinne, M., Ullrich, R., Scheibner, K., and Hofrichter, M. (2012). 'A spectrophotometric assay for the detection of fungal peroxygenases'. Anal. Biochem. 421, 327-329. doi: 10.1016/j.ab.2011.10.009

Poraj-Kobielska, M., Kinne, M., Ullrich, R., Scheibner, K., Kayser, G., Hammel, K. E., et al. (2011). 'Preparation of human drug metabolites using fungal peroxygenases'. Biochem. Pharmacol. 82, 789-796. doi: 10.1016/j.bcp.2011.06. 020

Poraj-Kobielska, M., Peter, S., Leonhardt, S., Ullrich, R., Scheibner, K., and Hofrichter, M. (2015). 'Immobilization of unspecific peroxygenases (EC 1.11.2.1) in PVA/PEG gel and hollow fiber modules'. Biochem. Eng. J. 98, 144-150. doi: 10.1016/j.bej.2015.02.037

Pourmir, A., and Johannes, T. W. (2012). Directed evolution: selection of the host organism. Comput. Struct. Biotechnol. J. 2:e201209012. doi: 10.5936/csbj. 201209012

Püllmann, P., Knorrscheidt, A., Münch, J., Palme, P. R., Hoehenwarter, W., Marillonnet, S., et al. (2021). A modular two yeast species secretion system for the production and preparative application of unspecific peroxygenases. Commun. Biol. 4:562. doi: 10.1038/s42003-021-02076-3

Püllmann, P., Ulpinnis, C., Marillonnet, S., Gruetzner, R., Neumann, S., and Weissenborn, M. J. (2019). Golden mutagenesis: an efficient multi-sitesaturation mutagenesis approach by Golden Gate cloning with automated primer design. Sci. Rep. 9:10932. doi: 10.1038/s41598-019-47376-1

Püllmann, P., and Weissenborn, M. J. (2021). Improving the heterologous production of fungal peroxygenases through an episomal Pichia pastoris promoter and signal peptide shuffling system. ACS Synth. Biol. doi: 10.1021/ acssynbio.0c00641 [Epub ahead of print].

Ramirez-Escudero, M., Molina-Espeja, P., Gómez de Santos, P., Hofrichter, M., Sanz-Aparicio, J., and Alcalde, M. (2018). 'Structural insights into the substrate promiscuity of a laboratory-evolved peroxygenase'. ACS Chem. Biol. 13, 32593268. doi: 10.1021/acschembio. 8 b00500

Ramirez-Ramirez, J., Martin-Diaz, J., Pastor, N., Alcalde, M., and Ayala, M. (2020). 'Exploring the role of phenylalanine residues in modulating the flexibility and topography of the active site in the peroxygenase variant PaDa-I'. Int. J. Mol. Sci. 21, 1-15. doi: 10.3390/ijms21165734

Reina, R., Liers, C., García-Romera, I., and Aranda, E. (2017). Enzymatic mechanisms and detoxification of dry olive-mill residue by Cyclocybe aegerita, Mycetinis alliaceus and Chondrostereum purpureum. Int. Biodeterior. Biodegradation 117:89. doi: 10.1016/j.ibiod.2016.11.029

Reymond, J.-L., Fluxà, V. S., and Maillard, N. (2009). Enzyme assays. Chem. Commun. 7, 34-46. doi: 10.1039/b813732c

Rolf, J., Rosenthal, K., and Lütz, S. (2019). Application of cell-free protein synthesis for faster biocatalyst development. Catalysts 9:190. doi: 10.3390/catal9020190

Rosenthal, K., and Lütz, S. (2018). 'Recent developments and challenges of biocatalytic processes in the pharmaceutical industry'. Curr. Opin. Green Sustain. Chem. 11, 58-64. doi: 10.1016/j.cogsc.2018.03.015

Rothschild-Mancinelli, K., Germann, S. M., and Andersen, M. R. (2020). "Bottlenecks and future outlooks for high-throughput technologies for filamentous fungi," in Grand Challenges in Fungal Biotechnology, ed. H. Nevalainen (Cham: Springer), 165-178. doi: 10.1007/978-3-030-29541-7_6 
Schmidt-Dannert, C., and Arnold, F. H. (1999). 'Directed evolution of industrial enzymes'. Trends Biotechnol. 17, 135-136. doi: 10.1016/S0167-7799(98)01 283-9

Schmitz, L., Rosenthal, K., and Lütz, S. (2017). "Enzyme-based electrobiotechnological synthesis," in Bioelectrosynthesis. Advances in Biochemical Engineering/Biotechnology, eds F. Harnisch and D. Holtmann (Cham: Springer), 87-134. doi: 10.1007/10_2017_33

Schmitz, L. M., Rosenthal, K., and Lütz, S. (2019a). Recent advances in heme biocatalysis engineering. Biotechnol. Bioeng. 116, 3469-3475. doi: 10.1002/bit. 27156

Schmitz, L. M., Schäper, J., Rosenthal, K., and Lütz, S. (2019b). 'Accessing the biocatalytic potential for $\mathrm{C}-\mathrm{H}$-activation by targeted genome mining and screening'. ChemCatChem 11, 5766-5777. doi: 10.1002/cctc.201901273

Sheldon, R. A. (2007). 'The E factor: fifteen years on'. Green Chem. 9, 1273-1283. doi: $10.1039 / \mathrm{b} 713736 \mathrm{~m}$

Sigmund, M.-C., and Poelarends, G. J. (2020). Current state and future perspectives of engineered and artificial peroxygenases for the oxyfunctionalization of organic molecules. Nat. Catal. 3, 690-702. doi: 10.1038/s41929-020-00507-8

Steinbrecht, S., Kiebist, J., König, R., Thiessen, M., Schmidtke, K. U., Kammerer, S., et al. (2020). Synthesis of cyclophosphamide metabolites by a peroxygenase from Marasmius rotula for toxicological studies on human cancer cells. $A M B$ Express 10:128. doi: 10.1186/s13568-020-01064-w

Sugano, S. S., Suzuki, H., Shimokita, E., Chiba, H., Noji, S., Osakabe, Y., et al. (2017). Genome editing in the mushroom-forming basidiomycete Coprinopsis cinerea, optimized by a high-throughput transformation system. Sci. Rep. 7:1260. doi: 10.1038/s41598-017-00883-5

Sundaramoorthy, M., Terner, J., and Poulos, T. L. (1995). 'The crystal structure of chloroperoxidase: a heme peroxidase-cytochrome P450 functional hybrid'. Structure 3, 1367-1378. doi: 10.1016/S0969-2126(01)00274-X

Tieves, F., Tonin, F., Fernández-Fueyo, E., Robbins, J. M., Bommarius, B., Bommarius, A. S., et al. (2019). 'Energising the E-factor: the E + -factor'. Tetrahedron 75, 1311-1314. doi: 10.1016/j.tet.2019.01.065

Ullrich, R., Dolge, C., Kluge, M., and Hofrichter, M. (2008). 'Pyridine as novel substrate for regioselective oxygenation with aromatic peroxygenase from Agrocybe aegerita'. FEBS Lett. 582, 4100-4106. doi: 10.1016/j.febslet.2008.11. 006

Ullrich, R., and Hofrichter, M. (2005). 'The haloperoxidase of the agaric fungus Agrocybe aegerita hydroxylates toluene and naphthalene'. FEBS Lett. 579, 6247-6250. doi: 10.1016/j.febslet.2005.10.014

Ullrich, R., and Hofrichter, M. (2007). 'Enzymatic hydroxylation of aromatic compounds'. Cell. Mol. Life Sci. 64, 271-293. doi: 10.1007/s00018-007-6362-1

Ullrich, R., Liers, C., Schimpke, S., and Hofrichter, M. (2009). 'Purification of homogeneous forms of fungal peroxygenase'. Biotechnol. J. 4, 1619-1626. doi: 10.1002/biot.200900076

Ullrich, R., Nueske, J., Scheibner, K., Spantzel, J., and Hofrichter, M. (2004). 'Novel haloperoxidase from the agaric basidiomycete Agrocybe aegerita oxidizes aryl alcohols and aldehydes'. Appl. Environ. Microbiol. 70, 4575-4581. doi: 10.1128/ AEM.70.8.4575

Ullrich, R., Poraj-Kobielska, M., Scholze, S., Halbout, C., Sandvoss, M., Pecyna, M. J., et al. (2018). 'Side chain removal from corticosteroids by unspecific peroxygenase’. J. Inorg. Biochem. 183, 84-93. doi: 10.1016/j.jinorgbio.2018.03. 011

Valderrama, B., Ayala, M., and Vazquez-Duhalt, R. (2002). 'Suicide inactivation of peroxidases and the challenge of engineering more robust enzymes'. Chem. Biol. 9, 555-565. doi: 10.1016/S1074-5521(02)00149-7

Vdovenko, M. M., Ullrich, R., Hofrichter, M., and Sakharov, I. Y. (2010). 'Luminol oxidation by hydrogen peroxide with chemiluminescent signal formation catalyzed by peroxygenase from the fungus Agrocybe aegerita V.Brig.'. Appl. Biochem. Microbiol. 46, 65-68. doi: 10.1134/S00036838100 10114

Vind, J., Østergaard, L.-H., De Maria, L., Kalum, L., and Amourgi, E. (2014). 'Peroxygenase Variants'. Patent (international): WO 2015/07 9064 A2

Wang, Y., Lan, D., Durrani, R., and Hollmann, F. (2017). Peroxygenases en route to becoming dream catalysts. What are the opportunities and challenges? Curr. Opin. Chem. Biol. 37, 1-9. doi: 10.1016/j.cbpa.2016.10.007

Yarman, A., Gröbe, G., Neumann, B., Kinne, M., Gajovic-Eichelmann, N., Wollenberger, U., et al. (2012). 'The aromatic peroxygenase from Marasmius rutola-a new enzyme for biosensor applications'. Anal. Bioanal. Chem. 402, 405-412. doi: 10.1007/s00216-011-5497-y

Yazbik, V., and Ansorge-Schumacher, M. (2010). 'Fast and efficient purification of chloroperoxidase from C. fumago’. Process Biochem. 45, 279-283. doi: 10.1016/ j.procbio.2009.09.006

Zhang, W., Burek, B. O., Fernández-Fueyo, E., Alcalde, M., Bloh, J. Z., and Hollmann, F. (2017). 'Selective activation of C-H bonds in a cascade process combining photochemistry and biocatalysis'. Angew. Chem. Int. Ed. Engl. 56, 15451-15455. doi: 10.1002/anie.201708668

Zhang, W., Li, H., Younes, S. H. H., Gómez de Santos, P., Tieves, F., Grogan, G., et al. (2021). 'Biocatalytic aromaticity-breaking epoxidation of naphthalene and nucleophilic ring-opening reactions'. ACS Catal. 11, 2644-2649. doi: 10.1021/ acscatal.0c05588

Conflict of Interest: The authors declare that the research was conducted in the absence of any commercial or financial relationships that could be construed as a potential conflict of interest.

Copyright (C) 2021 Kinner, Rosenthal and Lütz. This is an open-access article distributed under the terms of the Creative Commons Attribution License (CC BY). The use, distribution or reproduction in other forums is permitted, provided the original author(s) and the copyright owner(s) are credited and that the original publication in this journal is cited, in accordance with accepted academic practice. No use, distribution or reproduction is permitted which does not comply with these terms. 